\title{
LARGE SIEVE INEQUALITIES FOR QUARTIC CHARACTERS
}

\author{
PENG GAO AND LIANGYI ZHAO
}

\begin{abstract}
In this paper, we prove a large sieve inequality for quartic Dirichlet characters. The result is analogous to large sieve inequalities for the quadratic and cubic Dirichlet characters.
\end{abstract}

Mathematics Subject Classification (2010): 11L40, 11L99, 11T24

Keywords: large sieve, character sums, quartic Dirichlet characters

\section{INTRODUCTION}

The large sieve was an idea originated by J. V. Linnik [16] in 1941 while studying the distribution of quadratic non-residues. Refinements and extensions in various directions of this idea were made by many 11 9, 11, 12, 16, 18, 20, 21,23, 28. Large sieve results for Dirichlet characters with a fixed order are particularly useful in analytic number theory. We refer the readers to [10, Section 7, for some early large sieve-type results on general $r$-th order characters. Let $\left(a_{n}\right)_{n \in \mathbb{N}}$ be an arbitrary sequence of complex numbers, D. R. Heath-Brown's quadratic large sieve [12, Theorem 1] states that for any $\varepsilon>0$,

$$
\sum_{m \leq M}^{*}\left|\sum_{n \leq N}^{*} a_{n}\left(\frac{n}{m}\right)\right|^{2} \ll_{\varepsilon}(M N)^{\varepsilon}(M+N) \sum_{n \leq N}^{*}\left|a_{n}\right|^{2},
$$

where the asterisks indicate that $m, n$ run over positive odd square-free integers and $(\dot{\dot{m}})$ is the Jacobi symbol.

Similar to (1.1), Heath-Brown also established the following large sieve inequality involving the cubic symbols [13, Theorem 2]:

$$
\sum_{\substack{m \in \mathbb{Z}[\omega] \\ \mathcal{N}(m) \leq M}}^{*}\left|\sum_{\substack{n \in \mathbb{Z}[\omega] \\ \mathcal{N}(n) \leq N}}^{*} a_{n}\left(\frac{n}{m}\right)_{3}\right|^{2} \ll\left(M+N+(M N)^{2 / 3}\right)(M N)^{\varepsilon} \sum_{\mathcal{N}(n) \leq N}\left|a_{n}\right|^{2},
$$

where the asterisks indicate that $m, n$ run over square-free elements of $\mathbb{Z}[\omega], \omega=\exp (2 \pi i / 3)$ that are congruent to 1 modulo 3 and $(\dot{\bar{m}})_{3}$ is the cubic residue symbol. Moreover, here and after, we use $\mathcal{N}(m)$ to denote the norm of $m$.

Using (1.2), S. Baier and M. P. Young [3, Theorem 1.4] proved the following large sieve inequality for cubic Dirichlet characters:

$$
\begin{aligned}
& \sum_{Q<q \leq 2 Q} \sum_{\substack{\chi \bmod q \\
\chi^{3}=\chi_{0}}}^{\star}\left|\sum_{M<m \leq 2 M}^{*} a_{m} \chi(m)\right|^{2} \\
& \ll(Q M)^{\varepsilon} \min \left\{Q^{5 / 3}+M, Q^{4 / 3}+Q^{1 / 2} M, Q^{11 / 9}+Q^{2 / 3} M, Q+Q^{1 / 3} M^{5 / 3}+M^{12 / 5}\right\} \sum_{M<m \leq 2 M}^{*}\left|a_{m}\right|^{2},
\end{aligned}
$$

where the star on the sum over $\chi$ restricts the sum to primitive characters and the asterisks attached to the sum over $m$ indicates that $m$ runs over square-free integers.

Date: July 27, 2018. 
It is our goal in this paper to prove a large sieve inequality for quartic Dirichlet characters. First we prove the following theorem involving the quartic symbols.

Theorem 1.1. Let $M, N$ be positive integers, and let $\left(a_{n}\right)_{n \in \mathbb{N}}$ be an arbitrary sequence of complex numbers, where $n$ runs over $\mathbb{Z}[i]$. Then we have

$$
\sum_{\substack{m \in \mathbb{Z}[i] \\ \mathcal{N}(m) \leq M}}^{*}\left|\sum_{\substack{n \in \mathbb{Z}[i] \\ \mathcal{N}(n) \leq N}}^{*} a_{n}\left(\frac{n}{m}\right)_{4}\right|^{2} \ll_{\varepsilon}\left(M+N+(M N)^{3 / 4}\right)(M N)^{\varepsilon} \sum_{\mathcal{N}(n) \leq N}\left|a_{n}\right|^{2},
$$

for any $\varepsilon>0$, where the asterisks indicate that $m$ and $n$ run over square-free elements of $\mathbb{Z}[i]$ that are congruent to 1 modulo $(1+i)^{3}$ and $(\dot{\bar{m}})_{4}$ is the quartic residue symbol.

Next we shall establish the following large sieve inequality for quartic Dirichlet characters.

Theorem 1.2. Let $\left(a_{m}\right)_{m \in \mathbb{N}}$ be an arbitrary sequence of complex numbers. Then

$$
\begin{aligned}
& \sum_{Q<q \leq 2 Q} \sum_{\substack{\chi \bmod q \\
\chi^{4}=\chi_{0}, \chi^{2} \neq \chi_{0}}}^{\star}\left|\sum_{M<m \leq 2 M}^{*} a_{m} \chi(m)\right|^{2} \\
& \ll(Q M)^{\varepsilon} \min \left\{Q^{7 / 4}+M, Q^{11 / 8}+Q^{1 / 2} M, Q^{5 / 4}+Q^{2 / 3} M, Q+Q^{1 / 2} M+M^{17 / 7}\right\} \sum_{M<m \leq 2 M}^{*}\left|a_{m}\right|^{2},
\end{aligned}
$$

where the star on the sum over $\chi$ restricts the sum to primitive characters and the asterisks attached to the sum over $m$ indicates that $m$ runs over square-free integers.

Following the techniques of [12,13, Theorem 1.1] is proved via recursive uses of the Poisson summation formula. Theorem 1.2 follows, after some transformations, from Theorem 1.1 We note that (1.3) is used in (6.19). Mark that the characters involved in the second line of (6.19) are actually quadratic, since they are squares of the quartic symbol. Therefore, it is conceivable that the bounds in (1.4) can be improved if a large sieve inequality for quadratic characters in $\mathbb{Z}[i]$ is available.

Finally, we wish to mention that it is highly conceivable that these theorems will find applications in the study of families of $L$-functions involving quartic characters, analogous to those results in 3 and [17.

1.3. Notations. The following notations and conventions are used throughout the paper.

$e(z)=\exp (2 \pi i z)=e^{2 \pi i z}$.

$\widetilde{e}(z)=\exp (2 \pi i(z+\bar{z}))$.

$f=O(g)$ or $f \ll g$ means $|f| \leq c g$ for some unspecified positive constant $c$.

\section{Preliminaries}

2.1. Quartic symbol and the quartic Gauss sum. The symbol $(\dot{\bar{n}})_{4}$ is the quartic residue symbol in the ring $\mathbb{Z}[i]$. For a prime $\pi \in \mathbb{Z}[i]$ with $\mathcal{N}(\pi) \neq 2$, the quartic character is defined for $a \in \mathbb{Z}[i],(a, \pi)=1$ by $\left(\frac{a}{\pi}\right)_{4} \equiv a^{(\mathcal{N}(\pi)-1) / 4} \bmod \pi$, with $\left(\frac{a}{\pi}\right)_{4} \in\{ \pm 1, \pm i\}$. When $\pi \mid a$, it is defined that $\left(\frac{a}{\pi}\right)_{4}=0$. Then the quartic character can be extended to composite $n$ with $(\mathcal{N}(n), 2)=1$ multiplicatively.

Note that in $\mathbb{Z}[i]$, the ring of cosets modulo $(1+i)^{3}$ can be represented by $\{0, \pm 1, \pm i, 1+i, 2,2 i\}$ and every ideal coprime to 2 has a unique generator congruent to 1 modulo $(1+i)^{3}([15$, Lemma 7 , page 121]). Such a generator is called primary. Recall that the quartic reciprocity law states that for two primary primes $m, n \in \mathbb{Z}[i]$,

$$
\left(\frac{m}{n}\right)_{4}=\left(\frac{n}{m}\right)_{4}(-1)^{((\mathcal{N}(n)-1) / 4)((\mathcal{N}(m)-1) / 4)} .
$$

Observe that a non-unit $n=a+b i$ in $\mathbb{Z}[i]$ with $a, b \in \mathbb{Z}$ is congruent to 1 modulo $(1+i)^{3}$ if and only if $a \equiv 1 \bmod 4, b \equiv 0 \bmod 4$ or $a \equiv 3 \bmod 4, b \equiv 2 \bmod 4$ by Lemma 6 on page 121 of [15]. 
Below, we briefly discuss some properties of Gauss sums. These are dealt with in grater generality in Section 1 of [22]. For $n, r \in \mathbb{Z}[i], n \equiv 1 \bmod (1+i)^{3}$, we set

$$
g(r, n)=\sum_{x \bmod n}\left(\frac{x}{n}\right)_{4} \widetilde{e}\left(\frac{r x}{n}\right),
$$

where here and after

$$
\widetilde{e}(z)=\exp (2 \pi i(z+\bar{z})) .
$$

The quartic Gauss sum $g(n)$ is then defined to be $g(n)=g(1, n)$.

For $(s, n)=1$, we have

$$
g(r s, n)=\overline{\left(\frac{s}{n}\right)_{4}} g(r, n) .
$$

It's easy to see that the above equality in fact holds for any $s$ when $(\dot{\dot{n}})_{4}$ is a primitive character.

It's well-known that for square-free $n$ 's,

$$
|g(n)|=\sqrt{\mathcal{N}(n)}
$$

Suppose $n \equiv \pm 1 \bmod (1+i)^{3}$ with no rational prime divisor, so $(n, \bar{n})=1$. Let $\chi_{n}$ be a multiplicative character on $\mathbb{Z}[i] /(n)$, we define

$$
\tau\left(\chi_{n}\right)=\sum_{1 \leq x \leq \mathcal{N}(n)} \chi_{n}(x) e\left(\frac{x}{\mathcal{N}(n)}\right) .
$$

Now we specify $\chi_{n}$ to be $(\dot{\bar{n}})_{4}$. On writing $x=y \bar{n}+\bar{y} n$, where $y$ varies over a set of representatives in $\mathbb{Z}[i] \bmod n$, with $\bar{n}$ being the complex conjugate of $n$, it's easy to see that

$$
\tau\left(\chi_{n}\right)=\sum_{y \bmod n}\left(\frac{y \bar{n}}{n}\right)_{4} e\left(\frac{y}{n}+\frac{\bar{y}}{\bar{n}}\right)=\left(\frac{\bar{n}}{n}\right)_{4} g(n) .
$$

It follows that for $\left(n_{1}, n_{2}\right)=1$,

$$
\tau\left(\chi_{n_{1} n_{2}}\right)=\left(\frac{\mathcal{N}\left(n_{2}\right)}{n_{1}}\right)_{4}\left(\frac{\mathcal{N}\left(n_{1}\right)}{n_{2}}\right)_{4} \tau\left(\chi_{n_{1}}\right) \tau\left(\chi_{n_{2}}\right),
$$

and that if $n$ is square-free

$$
\left|\tau\left(\chi_{n}\right)\right|=\sqrt{\mathcal{N}(n)}
$$

Similarly, we have for $n$ square-free

$$
\left|\tau\left(\chi_{n}^{2}\right)\right|=\sqrt{\mathcal{N}(n)}
$$

2.2. Primitive quartic Dirichlet characters. The classification of all the primitive cubic characters of conductor $q$ coprime to 3 is given in [3. Similarly, one can give a classification of all the primitive quartic characters of conductor $q$ coprime to 2 . Every such character is of the form $m \rightarrow\left(\frac{m}{n}\right)_{4}$ for some $n \in \mathbb{Z}[i]$, with $n \equiv 1 \bmod (1+i)^{3}, n$ square-free and not divisible by any rational primes and $\mathcal{N}(n)=q$.

\section{Strategy for the Proof of Theorem 1.1}

Our proof of Theorem 1.1 uses the ideas in 12,13. We first estimate

$$
\sum_{1}=\left.\sum_{\substack{m \in \mathbb{Z}[i] \\ M<\mathcal{N}(m) \leq 2 M}}^{*} \sum_{\substack{n \in \mathbb{Z}[i] \\ N<\mathcal{N}(n) \leq 2 N}}^{*} a_{n}\left(\frac{n}{m}\right)_{4}\right|^{2} .
$$


We further simplify notation by supposing that the coefficients $a_{n}$ are supported on such integers $n \in \mathbb{Z}[i]$ satisfying $N<\mathcal{N}(n) \leq 2 N$. We begin by defining the norm

$$
\mathcal{B}_{1}(M, N)=\sup \left\{\sum_{1}: \sum_{n}\left|a_{n}\right|^{2}=1\right\} .
$$

Therefore, we need to show

$$
\mathcal{B}_{1}(M, N) \ll_{\varepsilon}(M N)^{\varepsilon}\left(M+N+(M N)^{3 / 4}\right) .
$$

Introducing a smooth weight function, we have

$$
\sum_{1} \ll \sum_{m} \exp \left(-2 \pi \frac{\mathcal{N}(m)}{M}\right)\left|\sum_{\substack{n \in \mathbb{Z}[i] \\ N<\mathcal{N}(n) \leq 2 N}}^{*} a_{n}\left(\frac{n}{m}\right)_{4}\right|^{2},
$$

the sum being over all $m \in \mathbb{Z}[i]$ for which $m \equiv 1 \bmod (1+i)^{3}$. If we now expand the above expression we obtain sums of the form

$$
\sum_{m} \exp \left(-2 \pi \frac{\mathcal{N}(m)}{M}\right)\left(\frac{n_{1}}{m}\right)_{4} \overline{\left(\frac{n_{2}}{m}\right)_{4}} .
$$

We note the following analogue of Lemma 2 of Heath-Brown and Patterson [14]. As the proof is similar, we omit it here.

Lemma 3.1. Let $\chi$ be a character of modulus $f \neq 1$, not necessarily primitive. Then, for $w \leq 1, \varepsilon>0$,

$$
\theta(w, \chi)=\sum_{\substack{a \equiv 1 \bmod (1+i)^{3} \\(a, f)=1}} \chi(a) e^{-2 \pi \mathcal{N}(a) w} \ll E(\chi) w^{-1}+\mathcal{N}(f)^{1 / 2+\varepsilon},
$$

where $E(\chi)=1$ if $\chi$ is principal, 0 otherwise. The implied constant depends only on $\varepsilon$.

Lemma 3.1 implies that each of these sums in (3.1) are $O\left(\mathcal{N}\left(n_{1} n_{2}\right)^{1 / 2+\varepsilon}\right)$, provided that the character involved is non-principal. Since $n_{1}$ and $n_{2}$ are square-free, $\left(\frac{n_{1}}{m}\right)_{4} \overline{\left(\frac{n_{2}}{m}\right)_{4}}$ is principle only if $n_{1}=n_{2}$. It follows that

$$
\sum_{1} \ll_{\varepsilon} N^{\varepsilon}\left(M \sum_{n}\left|a_{n}\right|^{2}+N \sum_{n_{1}, n_{2}}\left|a_{n_{1}} a_{n_{2}}\right|\right) \ll_{\varepsilon} N^{\varepsilon}\left(M+N^{2}\right) \sum_{n}\left|a_{n}\right|^{2} .
$$

We therefore have

$$
\mathcal{B}_{1}(M, N) \ll_{\varepsilon} N^{\varepsilon}\left(M+N^{2}\right) .
$$

This will be the starting point for an iterative bound for $\mathcal{B}_{1}(M, N)$.

Similar to the proof of [12, Lemma 1], using the duality principle (see for example, [19, Chap. 9]) and the quartic reciprocity law by considering the case for $n=a+b i$ with $a \equiv 1 \bmod 4, b \equiv 0 \bmod 4$ or $a \equiv 3 \bmod 4, b \equiv 2 \bmod 4$ (and similarly for $m$ ), we can establish the following lemma.

Lemma 3.2. We have $\mathcal{B}_{1}(M, N) \leq 2 \mathcal{B}_{1}(N, M)$. Moreover, there exist coefficients $a_{n}^{\prime}$, $a_{n}^{\prime \prime}$ with $\left|a_{n}^{\prime}\right|=\left|a_{n}^{\prime \prime}\right|=$ $\left|a_{n}\right|$ such that

$$
\begin{array}{r}
\sum_{\substack{m \in \mathbb{Z}[i] \\
M<\mathcal{N}(m) \leq 2 M}}^{*}\left|\sum_{\substack{n \in \mathbb{Z}[i] \\
N<\mathcal{N}(n) \leq 2 N}}^{*} a_{n}\left(\frac{n}{m}\right)_{4}\right|^{2} \leq 2 \sum_{\substack{m \in \mathbb{Z}[i] \\
M<\mathcal{N}(m) \leq 2 M}}^{*}\left|\sum_{\substack{n \in \mathbb{Z}[i] \\
N<\mathcal{N}(n) \leq 2 N}}^{*} a_{n}^{\prime}\left(\frac{m}{n}\right)_{4}\right|^{2} \\
\leq 4 \sum_{\substack{m \in \mathbb{Z}[i] \\
M<\mathcal{N}(m) \leq 2 M}}^{*}\left|\sum_{\substack{n \in \mathbb{Z}[i] \\
N<\mathcal{N}(n) \leq 2 N}}^{*} a_{n}^{\prime \prime}\left(\frac{n}{m}\right)_{4}\right|^{2}
\end{array}
$$


Our next lemma is a trivial modification of Lemma 9 of [12, which shows that the norm $\mathcal{B}_{1}(M, N)$ is essentially increasing.

Lemma 3.3. There is an absolute constant $C>0$ as follows. Let $M_{1}, N \geq 1$ and $M_{2} \geq C M_{1} \log \left(2 M_{1} N\right)$. Then

$$
\mathcal{B}_{1}\left(M_{1}, N\right) \leq C \mathcal{B}_{1}\left(M_{2}, N\right) .
$$

Similarly, if $M, N_{1} \geq 1$ and $N_{2} \geq C N_{1} \log \left(2 N_{1} M\right)$. Then

$$
\mathcal{B}_{1}\left(M, N_{1}\right) \leq C \mathcal{B}_{1}\left(M, N_{2}\right) .
$$

Next, we define

$$
\mathcal{B}_{2}(M, N)=\sup \left\{\sum_{2}: \sum_{n}\left|a_{n}\right|^{2}=1\right\} \text {, }
$$

where

$$
\sum_{2}=\sum_{\substack{m \in \mathbb{Z}[i] \\ M<\mathcal{N}(m) \leq 2 M}}\left|\sum_{\substack{n \in \mathbb{Z}[i] \\ N<\mathcal{N}(n) \leq 2 N}}^{*} a_{n}\left(\frac{m}{n}\right)_{4}\right|^{2},
$$

the summation over $m$ running over all integers of $\mathbb{Z}[i]$ in the relevant range.

It follows directly from Lemma 3.2 that

$$
\mathcal{B}_{1}(M, N) \leq 2 \mathcal{B}_{2}(M, N)
$$

For the other direction, we have the following.

Lemma 3.4. There exist $X, Y \gg 1$ such that $X Y^{3} \ll M$ and

$$
\mathcal{B}_{2}(M, N) \ll(\log M)^{3} M^{1 / 2} X^{-1 / 2} Y^{-3 / 2} \min \left(Y \mathcal{B}_{1}(X, N), X \mathcal{B}_{1}(Y, N)\right) .
$$

Proof. To handle $\sum_{2}$ we write each of the integers $m$ occurring in the outer summation of (3.3) in the form $m=a b^{2} c^{3} d$, where $a, b, c \equiv 1 \bmod (1+i)^{3}$ are square-free, and $d$ is a product of a unit, a power of $1+i$, and a fourth power (so that $d$ can be written as $d=u(1+i)^{j} e^{4}$ where $u$ is a unit, $0 \leq j \leq 3$ and $e \in \mathbb{Z}[i]$ ). We split the available ranges for $a, b, c$ and $d$ into sets $X<\mathcal{N}(a) \leq 2 X, Y<\mathcal{N}(b) \leq 2 Y, Z<\mathcal{N}(c) \leq 2 Z$ and $W<\mathcal{N}(d) \leq 2 W$, where $X, Y, Z$ and $W$ are powers of 2 . There will therefore be $O\left(\log ^{3} M\right)$ possible quadruples $X, Y, Z, W$. We may now write

$$
\sum_{2} \ll \sum_{X, Y, Z, W} \sum_{2}(X, Y, Z, W)
$$

accordingly, so that

$$
\sum_{2} \ll\left(\log ^{3} M\right) \sum_{2}(X, Y, Z, W)
$$

for some quadruple $X, Y, Z, W$. However,

$$
\sum_{2}(X, Y, Z, W) \leq \sum_{b, c, d} \sum_{\substack{a \in \mathbb{Z}[i] \\ X^{\prime}<\mathcal{N}(a) \leq 2 X^{\prime}}}^{*}\left|\sum_{\substack{n \in \mathbb{Z}[i] \\ N<\mathcal{N}(n) \leq 2 N}}^{*} a_{n}\left(\frac{b^{2} c^{3} d}{n}\right)_{4}\left(\frac{a}{n}\right)_{4}\right|^{2},
$$

where $X^{\prime}=X^{\prime}(b, c, d)=M / \mathcal{N}\left(b^{2} c^{3} d\right)$. It is easy to see that $X / 2 \leq X^{\prime} \leq 2 X$, and hence by Lemma 3.2

$$
\sum_{2}(X, Y, Z, W) \ll \sum_{b, c, d} \mathcal{B}_{1}\left(X^{\prime}, N\right) \sum_{n}\left|a_{n}\right|^{2} \ll Y Z W^{1 / 4} \max \left\{\mathcal{B}_{1}\left(X^{\prime}, N\right): X / 2 \leq X^{\prime} \leq 2 X\right\} \sum_{n}\left|a_{n}\right|^{2},
$$

since there are $O\left(W^{1 / 4}\right)$ possible integers $d$. 
In the same way we have

$$
\begin{aligned}
& \sum_{2}(X, Y, Z, W) \leq \sum_{a, b, d} \sum_{\substack{c \in \mathbb{Z}[i] \\
Z^{\prime}<\mathcal{N}(c) \leq 2 Z^{\prime}}}^{*} \sum_{\substack{n \in \mathbb{Z}[i] \\
N<\mathcal{N}(n) \leq 2 N}}^{*} a_{n}\left(\frac{a b^{2} d}{n}\right)_{4}\left(\frac{c^{3}}{n}\right)_{4}^{2} \\
& =\left.\sum_{a, b, d} \sum_{\substack{c \in \mathbb{Z}[i] \\
Z^{\prime}<\mathcal{N}(c) \leq 2 Z^{\prime}}}^{*} \sum_{\substack{n \in \mathbb{Z}[i] \\
N<\mathcal{N}(n) \leq 2 N}}^{*} \overline{a_{n}} \overline{\left(\frac{a b^{2} d}{n}\right)} \overline{\left(\frac{c^{3}}{n}\right)_{4}}\right|_{4} ^{2} \\
& =\left.\sum_{a, b, d} \sum_{\substack{c \in \mathbb{Z}[i] \\
Z^{\prime}<\mathcal{N}(c) \leq 2 Z^{\prime}}}^{*} \sum_{\substack{n \in \mathbb{Z}[i] \\
N<\mathcal{N}(n) \leq 2 N}}^{*} \bar{a}_{n} \overline{\left(\frac{a b^{2} d}{n}\right)_{4}}\left(\frac{c}{n}\right)_{4}\right|^{2} \\
& \ll \sum_{a, b, d} \mathcal{B}_{1}\left(Z^{\prime}, N\right) \sum_{n}\left|a_{n}\right|^{2} \\
& \ll X Y W^{1 / 4} \max \left\{\mathcal{B}_{1}\left(Z^{\prime}, N\right): Z \ll Z^{\prime} \ll Z\right\} \sum_{n}\left|a_{n}\right|^{2},
\end{aligned}
$$

where $Z^{\prime}=Z^{\prime}(a, b, d)=M / \mathcal{N}\left(a b^{2} d\right)$. As $Y \ll M^{1 / 2} X^{-1 / 2} Z^{-3 / 2} W^{-1 / 2}$, we see that

$$
\mathcal{B}_{2}(M, N) \ll(\log M)^{3} M^{1 / 2} X^{-1 / 2} Z^{-3 / 2} W^{-1 / 4} \min \left(Z \mathcal{B}_{1}(X, N), X \mathcal{B}_{1}(Z, N)\right) .
$$

The assertion of the lemma now follows on replacing $Z$ by $Y$ above.

As in [12], we introduce an infinitely differentiable weight function $W: \mathbb{R} \rightarrow \mathbb{R}$, defined by

$$
W(x)= \begin{cases}\exp \left(\frac{-1}{(2 x-1)(5-2 x)}\right), & \text { if } \frac{1}{2}<x<\frac{5}{2} \\ 0, & \text { otherwise }\end{cases}
$$

We now have

$$
\sum_{2} \ll \sum_{m \in \mathbb{Z}[i]} W\left(\frac{\mathcal{N}(m)}{M}\right)\left|\sum_{\substack{n \in \mathbb{Z}[i] \\ N<\mathcal{N}(n) \leq 2 N}} a_{n}\left(\frac{m}{n}\right)_{4}\right|^{2}
$$

where we recall that we can drop the conditions on $a_{n}$ on the inner sum above of the right-hand side expression by supposing that the coefficients $a_{n}$ are supported on square-free integers $n \equiv 1 \bmod (1+i)^{3} \in \mathbb{Z}[i]$ lying in the range $N<\mathcal{N}(n) \leq 2 N$.

Expanding the sum on the right-hand side of (3.6), we obtain

$$
\sum_{2} \ll \sum_{n_{1}, n_{2}} a_{n_{1}} \bar{a}_{n_{2}} \sum_{m \in \mathbb{Z}[i]} W\left(\frac{\mathcal{N}(m)}{M}\right)\left(\frac{m}{n_{1}}\right)_{4} \overline{\left(\frac{m}{n_{2}}\right)_{4}} .
$$

We set

$$
\sum_{3}=\sum_{3}(M, N)=\sum_{\left(n_{1}, n_{2}\right)=1} a_{n_{1}} \bar{a}_{n_{2}} \sum_{m \in \mathbb{Z}[i]} W\left(\frac{\mathcal{N}(m)}{M}\right)\left(\frac{m}{n_{1}}\right)_{4} \overline{\left(\frac{m}{n_{2}}\right)_{4}}
$$

and define

$$
\mathcal{B}_{3}(M, N)=\sup \left\{\sum_{3}: \sum_{n}\left|a_{n}\right|^{2}=1\right\} .
$$

Similar to [12, Lemma $7 ; 13$, we have the following 
Lemma 3.5. Let $\varepsilon>0$ be given. Then there exist positive integers $\Delta_{2} \geq \Delta_{1}$ such that

$$
\mathcal{B}_{2}(M, N) \ll_{\varepsilon} N^{\varepsilon} \mathcal{B}_{3}\left(\frac{M}{\Delta_{1}}, \frac{N}{\Delta_{2}}\right) .
$$

We complete the chain of relations amongst the various norms by giving the following estimate for $\mathcal{B}_{3}(M, N)$ in terms of $\mathcal{B}_{2}(M, N)$.

Lemma 3.6. Let $N \geq 1$. Then for any $\varepsilon>0$ we have

$$
\mathcal{B}_{3}(M, N) \ll_{\varepsilon} M N^{4 \varepsilon-1} \max \left\{\mathcal{B}_{2}(K, N): K \leq N^{2} / M\right\}+M^{-1} N^{3+4 \varepsilon} \sum_{K>N^{2} / M} K^{-2-\varepsilon} \mathcal{B}_{2}(K, N),
$$

where $K$ runs over powers of 2 .

This bound uses the Poisson summation formula and is the key in the proof of Theorem 1.1, Note that it does not cover the case in which $N=1 / 2$, say, for which we have the trivial bound

$$
\mathcal{B}_{3}(M, N) \ll_{\varepsilon} M, \quad(N \leq 1) .
$$

Section 4 will be devoted to the proof of Lemma 3.6

\section{Proof of Lemma 3.6}

Our proof of Lemma 3.6 requires the application of the Poisson summation formula. We shall write

$$
\chi(m)=\left(\frac{m}{n_{1}}\right)_{4} \overline{\left(\frac{m}{n_{2}}\right)_{4}},
$$

which is a primitive character (on the group $\left(\mathbb{Z}[i] /\left(n_{1} n_{2}\right)\right)^{\times}$) to modulus $q=n_{1} n_{2}$, provided that $n_{1}, n_{2}$ and 2 are pair-wise coprime and that $n_{1}$ and $n_{2}$ are square-free.

Lemma 4.1. With the above notations we have

$$
\sum_{m \in \mathbb{Z}[i]} W\left(\frac{\mathcal{N}(m)}{M}\right) \chi(m)=\frac{\chi(-2 i) g\left(n_{1}\right) \overline{g\left(n_{2}\right)} M}{\mathcal{N}(q)}\left(\frac{n_{2}}{n_{1}}\right)_{4} \overline{\left(\frac{n_{1}}{n_{2}}\right)_{4}}\left(\frac{-1}{n_{2}}\right)_{4} \sum_{m \in \mathbb{Z}[i]} \widetilde{W}\left(\sqrt{\frac{\mathcal{N}(k) M}{\mathcal{N}(q)}}\right) \bar{\chi}(k),
$$

where

$$
\widetilde{W}(t)=\int_{-\infty}^{\infty} \int_{-\infty}^{\infty} W(\mathcal{N}(x+y i)) \widetilde{e}\left(\frac{t(x+y i)}{2 i}\right) \mathrm{d} x \mathrm{~d} y
$$

for non-negative $t$. Here $\widetilde{e}(z)$ is defined in (2.1) and $g(n)$ is the Gauss sums defined in Section [2.1.

Proof. This lemma is analogous to Lemma 10 in 13 and the proof is very similar. The differences include we need to start with the Poisson summation formula for $\mathbb{Z}[i]$, which takes the form.

$$
\sum_{j \in \mathbb{Z}[i]} f(j)=\sum_{k \in \mathbb{Z}[i]} \int_{-\infty}^{\infty} \int_{-\infty}^{\infty} f(x+y i) \widetilde{e}\left(\frac{k(x+y i)}{2 i}\right) \mathrm{d} x \mathrm{~d} y .
$$

We omit the details of the rest of proof as it simply goes along the same lines as the proof of Lemma 10 in [13. [12.

Our next result will be used to separate the variables in a function of a product, which is Lemma 12 of

Lemma 4.2. Let $\rho: \mathbb{R} \rightarrow \mathbb{R}$ be an infinitely differentiable function whose derivatives satisfy the bound

$$
\rho(k)(x) \ll_{k, A}|x|^{-A}
$$

for $|x| \geq 1$, for any positive constant $A$. Let

$$
\rho_{+}(s)=\int_{0}^{\infty} \rho(x) x^{s-1} \mathrm{~d} x, \quad \rho_{-}(s)=\int_{0}^{\infty} \rho(-x) x^{s-1} \mathrm{~d} x .
$$


Then $\rho_{+}(s)$ and $\rho_{-}(s)$ are holomorphic in $\Re(s)=\sigma>0$, and satisfy

$$
\rho_{+}(s), \rho_{-}(s) \ll_{A, \sigma}|s|^{-A},
$$

in that same domain, for any positive constant $A$. Moreover if $\sigma>0$ we have

$$
\rho(x)=\frac{1}{2 \pi i} \int_{\sigma-i \infty}^{\sigma+i \infty} \rho_{+}(s) x^{-s} \mathrm{~d} s \text { and } \rho(-x)=\frac{1}{2 \pi i} \int_{\sigma-i \infty}^{\sigma+i \infty} \rho_{-}(s) x^{-s} \mathrm{~d} s
$$

for any positive $x$.

We are now ready to prove Lemma 3.6 .

Proof of Lemma 3.6. In the notation of Lemma 4.1 we have

$$
\sum_{3}(M, N)=\sum_{\left(n_{1}, n_{2}\right)=1} a_{n_{1}} \bar{a}_{n_{2}} \sum_{m \in \mathbb{Z}[i]} W\left(\frac{\mathcal{N}(m)}{M}\right) \chi(m) .
$$

We proceed to evaluate the inner sum using Lemma 4.1, whence

$$
\sum_{3}(M, N)=M \sum_{k \in \mathbb{Z}[i]} \sum_{\left(n_{1}, n_{2}\right)=1} c_{n_{1}} \bar{c}_{n_{2}}\left(\frac{n_{2}}{n_{1}}\right)_{4} \overline{\left(\frac{n_{1}}{n_{2}}\right)_{4}}\left(\frac{-1}{n_{2}}\right)_{4} \widetilde{W}\left(\sqrt{\frac{\mathcal{N}(k) M}{\mathcal{N}\left(n_{1} n_{2}\right)}}\right) \bar{\chi}(k),
$$

where

$$
c_{n}=a_{n}\left(\frac{-2 i}{n}\right)_{4} \frac{g(n)}{\mathcal{N}(n)} .
$$

Note by the law of quartic reciprocity, we have

$$
\left(\frac{n_{2}}{n_{1}}\right)_{4} \overline{\left(\frac{n_{1}}{n_{2}}\right)_{4}}=(-1)^{\left(\left(\mathcal{N}\left(n_{1}\right)-1\right) / 4\right)\left(\left(\mathcal{N}\left(n_{2}\right)-1\right) / 4\right)} .
$$

Now we let

$$
S_{1}=\{n \in \mathbb{Z}[i]: N<\mathcal{N}(n) \leq 2 N, n \text { square-free, } n=a+b i, a, b \in \mathbb{Z}, a \equiv 1 \bmod 4, b \equiv 0 \bmod 4\},
$$

and

$$
S_{2}=\{n \in \mathbb{Z}[i]: N<\mathcal{N}(n) \leq 2 N, n \text { square-free, } n=a+b i, a, b \in \mathbb{Z}, a \equiv 3 \bmod 4, b \equiv 2 \bmod 4\} .
$$

We can then recast the inner sum in (4.1) as

$$
\begin{aligned}
& \sum_{\substack{\left(n_{1}, n_{2}\right)=1 \\
=}} \sum_{\substack{\left(n_{1}, n_{2}\right)=1 \\
n_{1} \in S_{1}, n_{2} \in S_{1}}} \cdots+\sum_{\substack{\left(n_{1}, n_{2}\right)=1 \\
n_{1} \in S_{1}, n_{2} \in S_{2}}} \cdots+\sum_{\substack{\left(n_{1}, n_{2}\right)=1 \\
n_{1} \in S_{2}, n_{2} \in S_{1}}} \cdots+\sum_{\substack{\left(n_{1}, n_{2}\right)=1 \\
n_{1} \in S_{2}, n_{2} \in S_{2}}} \cdots-2 \sum_{\substack{\left(n_{1}, n_{2}\right)=1 \\
n_{1} \in S_{2}, n_{2} \in S_{2}}} \cdots \\
= & \sum_{\left(n_{1}, n_{2}\right)=1} c_{n_{1}} \bar{c}_{n_{2}}\left(\frac{-1}{n_{2}}\right)_{4} \widetilde{W}\left(\sqrt{\frac{\mathcal{N}(k) M}{\mathcal{N}\left(n_{1} n_{2}\right)}}\right) \bar{\chi}(k)-2 \sum_{\left(n_{1}, n_{2}\right)=1} c_{n_{1}}^{\prime} \bar{c}^{\prime} n_{2}\left(\frac{-1}{n_{2}}\right)_{4} \widetilde{W}\left(\sqrt{\frac{\mathcal{N}(k) M}{\mathcal{N}\left(n_{1} n_{2}\right)}}\right) \bar{\chi}(k),
\end{aligned}
$$

where we let $c_{n}^{\prime}=c_{n}$ if $n \in S_{2}$ and 0 otherwise. Due to similarities, it suffices to estimate

$$
M \sum_{k \in \mathbb{Z}[i]} \sum_{\left(n_{1}, n_{2}\right)=1} c_{n_{1}} \bar{c}_{n_{2}}\left(\frac{-1}{n_{2}}\right)_{4} \widetilde{W}\left(\sqrt{\frac{\mathcal{N}(k) M}{\mathcal{N}\left(n_{1} n_{2}\right)}}\right) \bar{\chi}(k),
$$

Note that $k=0$ may be omitted if $N \geq 1$, since then $\mathcal{N}\left(n_{1} n_{2}\right)>1$ and $\chi(0)=0$, the character being non-trivial. We may now apply Lemma 4.2 to the function $\rho(x)=\widetilde{W}(x)$, which satisfies the necessary conditions of the lemma, as one sees by repeated integration by parts. We decompose the available $k$ into sets for which $K<\mathcal{N}(k) \leq 2 K$, where $K$ runs over powers of 2 , and use

$$
\sigma= \begin{cases}\varepsilon, & \text { for } K \leq N^{2} / M \\ 4+\varepsilon, & \text { otherwise }\end{cases}
$$


This gives

$$
\sum_{3} \ll_{\varepsilon} M \sum_{K}(K M)^{-\sigma / 2} \int_{-\infty}^{\infty}\left|\rho_{+}(\sigma+i t)\right||S(\sigma+i t)| \mathrm{d} t
$$

where

$$
S(s)=\sum_{K<\mathcal{N}(k) \leq 2 K}\left|\sum_{\left(n_{1}, n_{2}\right)=1} b_{n_{1}} b_{n_{2}}^{\prime}\left(\frac{-1}{n_{2}}\right)_{4} \bar{\chi}(k)\right|, \quad \text { with } b_{n}=c_{n} \mathcal{N}(n)^{s / 2} \text { and } b_{n}^{\prime}=\bar{c}_{n} \mathcal{N}(n)^{s / 2} .
$$

We use the Möbius function to detect the coprimality condition in the inner sum of $S(s)$, giving

$$
\begin{aligned}
S(s) & \ll \sum_{d} \sum_{K<\mathcal{N}(k) \leq 2 K}\left|\sum_{d \mid\left(n_{1}, n_{2}\right)} b_{n_{1}} b_{n_{2}}^{\prime}\left(\frac{-1}{n_{2}}\right)_{4} \bar{\chi}(k)\right| \\
& =\sum_{d} \sum_{K<\mathcal{N}(k) \leq 2 K}\left|\sum_{d \mid n} b_{n} \overline{\left(\frac{k}{n}\right)_{4}}\right|\left|\sum_{d \mid n} b_{n}^{\prime}\left(\frac{-k}{n}\right)_{4}\right| \leq S_{1}^{1 / 2} S_{2}^{1 / 2},
\end{aligned}
$$

by Cauchy's inequality, where

$$
S_{1}=\sum_{d} \sum_{K<\mathcal{N}(k) \leq 2 K}\left|\sum_{d \mid n} b_{n} \overline{\left(\frac{k}{n}\right)}\right|_{4}^{2}
$$

and satisfies the bound

$$
S_{1} \leq \sum_{d} \mathcal{B}_{2}(K, N) \sum_{d \mid n}\left|b_{n}\right|^{2} \leq \mathcal{B}_{2}(K, N) \sum_{n} d(n)\left|a_{n}\right|^{2} \mathcal{N}(n)^{\sigma-1} \ll_{\varepsilon} N^{\varepsilon+\sigma-1} \mathcal{B}_{2}(K, N) .
$$

$S_{2}$ can be treated similarly. It follows then that

$$
S(s) \ll_{\varepsilon} N^{\varepsilon+\sigma-1} \mathcal{B}_{2}(K, N),
$$

and since

$$
\int_{-\infty}^{\infty}\left|\rho_{+}(\sigma+i t)\right| \mathrm{d} t \ll_{\varepsilon} 1
$$

we infer, mindful of our choices of $\sigma$ in (4.2), that

$$
\sum_{3} \ll_{\varepsilon} M N^{4 \varepsilon-1} \max \left\{\mathcal{B}_{2}(K, N): K \leq N^{2} / M\right\}+M^{-1} N^{3+4 \varepsilon} \sum_{K>N^{2} / M} K^{-2-\varepsilon} \mathcal{B}_{2}(K, N),
$$

Recalling the definition of $\mathcal{B}_{3}(M, N)$ in (3.7), we have completed the proof of Lemma 3.6.

\section{The Recursive Estimate and the Proof of Theorem 1.1}

Lemmas 3.4, 3.5 and 3.6 allow us to estimate $\mathcal{B}_{1}(M, N)$ recursively, as follows.

Lemma 5.1. Suppose that $3 / 2<\xi \leq 2$, and that

$$
\mathcal{B}_{1}(M, N) \ll_{\varepsilon}(M N)^{\varepsilon}\left(M+N^{\xi}+(M N)^{3 / 4}\right) .
$$

for any $\varepsilon>0$. Then

$$
\mathcal{B}_{1}(M, N) \ll_{\varepsilon}(M N)^{\varepsilon}\left(M+N^{(9 \xi-6) /(4 \xi-1)}+(M N)^{3 / 4}\right) .
$$

for any $\varepsilon>0$. 
Proof. By the symmetry expressed in Lemma 3.2 the hypothesis (5.1) yields

$$
\mathcal{B}_{1}(M, N) \ll_{\varepsilon}\left(M^{\xi}+N+(M N)^{3 / 4}\right)(M N)^{\varepsilon} .
$$

It follows from (3.2) that the above estimation is valid with $\xi=2$. We now feed this into Lemma 3.4. whence

$$
\mathcal{B}_{2}(M, N) \ll_{\varepsilon}(M N)^{2 \varepsilon} M^{1 / 2} X^{-1 / 2} Y^{-3 / 2} \min (Y f(X, N), X f(Y, N)),
$$

where

$$
f(Z, N)=Z^{\xi}+N+(Z N)^{3 / 4} .
$$

If $X \geq Y$ we bound the minimum in (5.2) by $Y f(X, N)$, whence

$$
\mathcal{B}_{2}(M, N) \ll_{\varepsilon}(M N)^{2 \varepsilon} M^{1 / 2} X^{-1 / 2} Y^{-3 / 2}\left(Y X^{\xi}+Y N+Y(X N)^{3 / 4}\right) .
$$

Here we have

$$
M^{1 / 2} X^{-1 / 2} Y^{-3 / 2} Y X^{\xi} \ll M^{\xi} Y^{1-3 \xi}
$$

since $X \ll M Y^{-3}$. On recalling that $\xi>3 / 2>1 / 3$ and $Y \gg 1$ we see that this is $O\left(M^{\xi}\right)$. Moreover

$$
M^{1 / 2} X^{-1 / 2} Y^{-3 / 2} Y N=M^{1 / 2} X^{-1 / 2} Y^{-1 / 2} N \ll M^{1 / 2} N .
$$

Finally

$$
M^{1 / 2} X^{-1 / 2} Y^{-3 / 2} Y(X N)^{3 / 4}=M^{1 / 2} X^{1 / 4} Y^{-1 / 2} N^{3 / 4} \ll M^{3 / 4} N^{3 / 4} \ll M^{1 / 2} N+M^{3 / 2} \leq M^{1 / 2} N+M^{\xi},
$$

since $\xi>3 / 2$. It follows that

$$
\mathcal{B}_{2}(M, N) \ll_{\varepsilon}(M N)^{2 \varepsilon}\left(M^{1 / 2} N+M^{\xi}\right)
$$

when $X \geq Y$.

In the alternative case we bound the minimum in (5.2) by $X f(Y, N)$, whence

$$
\mathcal{B}_{2}(M, N) \ll_{\varepsilon}(M N)^{2 \varepsilon} M^{1 / 2} X^{-1 / 2} Y^{-3 / 2}\left(X Y^{\xi}+X N+X(Y N)^{3 / 4}\right) .
$$

Here

$$
M^{1 / 2} X^{-1 / 2} Y^{-3 / 2} X Y^{\xi} \ll M^{1 / 2} X^{1 / 2} Y^{1 / 2} \ll M \ll M^{\xi}
$$

since $\xi \leq 2$ and $X Y \ll M$. Moreover

$$
M^{1 / 2} X^{-1 / 2} Y^{-3 / 2} X N=M^{1 / 2} X^{1 / 2} Y^{-3 / 2} N \ll M^{1 / 2} N
$$

since we are now supposing that $Y \geq X$. Finally

$$
M^{1 / 2} X^{-1 / 2} Y^{-3 / 2} X(Y N)^{3 / 4}=M^{1 / 2} X^{1 / 2} Y^{-3 / 4} N^{3 / 4} \ll M^{1 / 2} Y^{-1 / 4} N^{3 / 4} \ll M^{1 / 2} N^{3 / 4} \ll M^{1 / 2} N+M^{\xi},
$$

as before. It follows that (5.3) holds when $Y \geq X$ too. It will be convenient to observe that (5.3) still holds when $M<1 / 2$, since then $\mathcal{B}_{2}(M, N)=0$.

We are now ready to use (5.3) (with a new value for $\varepsilon$ ) in Lemma 3.6, to obtain a bound for $\mathcal{B}_{3}(M, N)$. We readily see that

$$
\max \left\{\mathcal{B}_{2}(K, N): K \leq N^{2} / M\right\} \ll_{\varepsilon} N^{\varepsilon}\left(M^{-1 / 2} N^{2}+M^{-\xi} N^{2 \xi}\right)
$$

and

$$
\sum_{K>N^{2} / M} K^{-2-\varepsilon} \mathcal{B}_{2}(K, N) \ll_{\varepsilon} N^{\varepsilon}\left(M^{3 / 2} N^{-2}+M^{2-\xi} N^{2 \xi-4}\right) .
$$

Thus, if $N \geq 1$, we will have

$$
\mathcal{B}_{3}(M, N) \ll_{\varepsilon} N^{5 \varepsilon}\left(M^{1 / 2} N+M^{1-\xi} N^{2 \xi-1}\right) .
$$


When this is used in Lemma 3.5 we find that when $N / \Delta_{2} \geq 1$,

$$
\begin{aligned}
\mathcal{B}_{3}\left(\frac{M}{\Delta_{1}}, \frac{N}{\Delta_{2}}\right) & \ll_{\varepsilon} N^{5 \varepsilon}\left(M^{1 / 2} N+M^{1-\xi} N^{2 \xi-1} \Delta_{1}^{\xi-1} \Delta_{2}^{1-2 \xi}\right) \leq N^{5 \varepsilon}\left(M^{1 / 2} N+M^{1-\xi} N^{2 \xi-1} \Delta_{2}^{-\xi}\right) \\
& \leq N^{5 \varepsilon}\left(M^{1 / 2} N+M^{1-\xi} N^{2 \xi-1}\right) .
\end{aligned}
$$

Note that when $M \geq N$, we have $M^{1 / 2} N \leq(M N)^{3 / 4}$ and when $M \leq N$, we have $(M N)^{3 / 4} \leq M^{1-\xi} N^{2 \xi-1}$ since $\xi>3 / 2$. Thus we conclude that

$$
\mathcal{B}_{2}(M, N) \ll_{\varepsilon} N^{6 \varepsilon}\left((M N)^{3 / 4}+M^{1-\xi} N^{2 \xi-1}\right),
$$

provided that $N / \Delta_{2} \geq 1$. In the alternative case (3.8) applies, whence

$$
\mathcal{B}_{2}(M, N) \ll_{\varepsilon}(M N)^{6 \varepsilon}\left(M+(M N)^{3 / 4}+M^{1-\xi} N^{2 \xi-1}\right),
$$

In view of Lemma 3.3 and (3.4) we may now deduce that

$$
\mathcal{B}_{1}(M, N) \leq \mathcal{B}_{1}\left(M^{\prime}, N\right) \ll \mathcal{B}_{2}\left(M^{\prime}, N\right) \ll_{\varepsilon}\left(M^{\prime} N\right)^{6 \varepsilon}\left(M^{\prime}+\left(M^{\prime} N\right)^{3 / 4}+M^{\prime 1-\xi} N^{2 \xi-1}\right),
$$

for any $M^{\prime} \geq C M \log (2 M N)$. Note that when $M^{4 \xi-1} \leq N^{8 \xi-7}$, we have

$$
(M N)^{3 / 4} \leq M^{1-\xi} N^{2 \xi-1} \text {. }
$$

We shall now choose

$$
M^{\prime}=C \max \left\{M, N^{(8 \xi-7) /(4 \xi-1)}\right\} \log (2 M N),
$$

so that when $M \geq N^{(8 \xi-7) /(4 \xi-1)}$, we have

$$
M^{\prime}+\left(M^{\prime} N\right)^{3 / 4}+M^{\prime 1-\xi} N^{2 \xi-1} \ll(M N)^{\varepsilon}\left(M+(M N)^{3 / 4}\right),
$$

while when $M \leq N^{(8 \xi-7) /(4 \xi-1)}$, we have

$$
\begin{aligned}
M^{\prime}+\left(M^{\prime} N\right)^{3 / 4}+M^{\prime 1-\xi} N^{2 \xi-1} & \ll(M N)^{\varepsilon}\left(N^{(8 \xi-7) /(4 \xi-1)}+N^{(8 \xi-7)(1-\xi) /(4 \xi-1)} N^{2 \xi-1}\right) \\
& \ll(M N)^{\varepsilon} N^{(9 \xi-6) /(4 \xi-1)} .
\end{aligned}
$$

We then deduce that

$$
\mathcal{B}_{1}(M, N) \ll_{\varepsilon}(M N)^{20 \varepsilon}\left(M+(M N)^{3 / 4}+N^{(9 \xi-6) /(4 \xi-1)}\right) .
$$

Lemma 5.1 now follows.

We now proceed to prove Theorem 1.1.

Proof of Theorem 1.1. Note that it follows from (3.2) that the estimation given in Lemma 5.1 is valid with $\xi=2$. We further observe that

$$
\frac{3}{2}<\frac{9 \xi-6}{4 \xi-1}<\xi
$$

for $\xi>3 / 2$ and in the iterative applications of Lemma 5.1 the exponent of $N$ in the bound for $\mathcal{B}_{1}(M, N)$ decreases and tends to $3 / 2$. We therefore arrive at the following bound

$$
\mathcal{B}_{1}(M, N) \ll_{\varepsilon}(M N)^{\varepsilon}\left(M+N^{3 / 2}+(M N)^{3 / 4}\right),
$$

for any $\varepsilon>0$. Using Lemma 3.2 we then have

$$
\begin{aligned}
\mathcal{B}_{1}(M, N) & \ll_{\varepsilon}(M N)^{\varepsilon} \min \left\{M+N^{3 / 2}+(M N)^{3 / 4}, N+M^{3 / 2}+(M N)^{3 / 4}\right\} \\
& \ll_{\varepsilon}(M N)^{\varepsilon}\left(M+N+(M N)^{3 / 4}\right),
\end{aligned}
$$

where the last estimation follows since when $N \leq M, N^{3 / 2}=N^{3 / 4} N^{3 / 4} \leq(M N)^{3 / 4}$ and similarly when $N \geq M, M^{3 / 2} \leq(M N)^{3 / 4}$. This establishes Theorem 1.1. 


\section{The Quartic large sieve for Dirichlet Characters}

We now proceed to prove Theorem 1.2, It is easy to reduce the expression on the left-hand side of (1.4) to a sum of similar expressions with the additional summation conditions $(q, 2)=1$ and $(m, 2)=1$ included. Thus it suffices to estimate

$$
\begin{aligned}
\sum_{\substack{Q<q \leq 2 Q \\
(q, 2)=1}} \sum_{\substack{\chi \bmod q \\
\chi^{4}=\chi_{0}, \chi^{2} \neq \chi_{0}}}^{\star}\left|\sum_{\substack{M<m \leq 2 M \\
(m, 2)=1}}^{*} a_{m} \chi(m)\right|^{2} & =\sum_{\substack{n \in \mathbb{Z}[i] \\
Q<\mathcal{N}(n) \leq 2 Q \\
n \equiv 1 \bmod (1+i)^{3}}}^{\prime}\left|\sum_{\substack{M<m \leq 2 M \\
(m, 2)=1}}^{*} a_{m} \chi_{n}(m)\right|^{2} \\
& =\frac{1}{2} \sum_{\substack{n \in \mathbb{Z}[i] \\
Q<\mathcal{N}(n) \leq 2 Q \\
n \equiv \pm 1 \bmod (1+i)^{3}}}^{\prime}\left|\sum_{\substack{M<m \leq 2 M \\
(m, 2)=1}}^{*} a_{m} \chi_{n}(m)\right|^{2},
\end{aligned}
$$

where the apostrophe indicates that $n$ is square-free and has no rational prime divisor and $\chi_{n}(m)=\left(\frac{m}{n}\right)_{4}$ is the quartic residue symbol. We shall use this notation for all $n \in \mathbb{Z}[i]$ and $m \in \mathbb{Z}$.

6.1. Definition of certain norms. In the following, we shall estimate the expression in the last line of (6.1). We begin by defining a norm corresponding to the double sum in the last line of (6.1) by

$$
B_{1}(Q, M):=\sup _{\left(a_{m}\right)}\left\|a_{m}\right\|^{-2} \sum_{\substack{n \in \mathbb{Z}[i] \\ Q<\mathcal{N}(n) \leq 2 Q \\ n \equiv \pm 1 \bmod (1+i)^{3}}}^{\prime}\left|\sum_{\substack{M<m \leq 2 M \\(m, 2)=1}}^{*} a_{m} \chi_{m}(n)\right|^{2},
$$

where

$$
\left\|a_{m}\right\|^{2}=\sum_{m}\left|a_{m}\right|^{2}
$$

and where by convention we suppose that $\left(a_{m}\right)$ is not identically zero.

We further define a norm $B_{2}(Q, M)$ in the same way as $B_{1}(Q, M)$ except removing the condition that $n$ has no rational prime divisor. Similarly, we define a norm $B_{3}(Q, M)$ by further removing the condition that $n$ is square-free.

We now use the function $W(x)$ defined in (3.5) to see that

$$
B_{3}(Q, M) \ll \sup _{\left(a_{m}\right)}\left\|a_{m}\right\|^{-2} \sum_{n \in \mathbb{Z}[i]} W\left(\frac{\mathcal{N}(n)}{Q}\right)\left|\sum_{\substack{M<m \leq 2 M \\(m, 2)=1}}^{*} a_{m} \chi_{m}(n)\right|^{2} .
$$

Expanding the sum on the right-hand side, we obtain

$$
\sum_{\substack{M<m_{1} \leq 2 M \\ M<m_{2} \leq 2 M \\\left(m_{1} m_{2}, 2\right)=1}}^{*} a_{m_{1}} \bar{a}_{m_{2}} \sum_{n \in \mathbb{Z}[i]} W\left(\frac{\mathcal{N}(n)}{Q}\right) \chi_{m_{1}}(n) \bar{\chi}_{m_{2}}(n) .
$$

As in [12, it turns out that it suffices to restrict our attention to the case in which $m_{1}$ and $m_{2}$ are coprime. To see this, we sort the terms in (6.2) according to $\delta=\left(m_{1}, m_{2}\right)$, and detecting the condition $(n, \delta)=1$ by 
the Möbius function for $\mathbb{Z}[i]$, we obtain that the expression in (6.2) equals

$$
\begin{aligned}
\sum_{(\delta, 2)=1}^{*} \sum_{d \mid \delta} \mu_{[i]}(d) \sum_{s \in \mathbb{Z}[i]} W\left(\frac{\mathcal{N}(s)}{Q / \mathcal{N}(d)}\right) & \sum_{\substack{M / \delta<r_{1} \leq 2 M / \delta \\
M / \delta<r_{2} \leq 2 M / \delta \\
\left(r_{1} r_{2}, 2 \delta\right)=1 \\
\left(r_{1}, r_{2}\right)=1}}^{*} a_{r_{1}}^{*} \overline{a_{r_{2}}^{*}} \chi_{r_{1}}(s) \overline{\chi_{r_{2}}}(s) \\
& \ll \sum_{(\delta, 2)=1}^{*} \sum_{d \mid \delta} B_{4}\left(\frac{Q}{\mathcal{N}(d)}, \frac{M}{\delta}\right) \sum_{\substack{M / \delta<r<2 M / \delta \\
(r, 2 \delta)=1}}^{*}\left|a_{r \delta}\right|^{2},
\end{aligned}
$$

where $d$ runs over non-associate divisors of $\delta$ in $\mathbb{Z}[i]$,

$$
a_{r}^{*}:=a_{r \delta} \chi_{r}(d),
$$

and

$$
B_{4}(Q, M):=\sup _{\left(a_{m}\right)}\left\|a_{m}\right\|^{-2} \sum_{\substack{M<m_{1}, m_{2} \leq 2 M \\\left(m_{1} m_{2}, 2\right)=1 \\\left(m_{1}, m_{2}\right)=1}}^{*} a_{m_{1}} \bar{a}_{m_{2}} \sum_{n \in \mathbb{Z}[i]} W\left(\frac{\mathcal{N}(n)}{Q}\right) \chi_{m_{1}}(n) \bar{\chi}_{m_{2}}(n) .
$$

Moreover, we define a norm $C_{1}(M, Q)$ dual to $B_{1}(Q, M)$ by

$$
C_{1}(M, Q):=\sup _{\left(b_{n}\right)}\left\|b_{n}\right\|^{-2} \sum_{\substack{M<m \leq 2 M \\(m, 2)=1}}^{*}\left|\sum_{\substack{n \in \mathbb{Z}[i] \\ Q<\mathcal{N}(n) \leq 2 Q \\ n \equiv \pm 1 \bmod (1+i)^{3}}}^{\prime} b_{n} \chi_{n}(m)\right|^{2} .
$$

By the duality principle, we have

$$
B_{1}(Q, M)=C_{1}(M, Q) .
$$

Furthermore, we define a norm $C_{2}(M, Q)$ by extending the summation over $m$ in the definition of $C_{1}(M, Q)$ to all integers $m$ with $M<m \leq 2 M$. Trivially, we have

$$
C_{1}(M, Q) \leq C_{2}(M, Q) .
$$

6.2. Comparison of the norms. For the proof of our Theorem 1.2, we need the following lemma on the norms defined in the previous section.

Lemma 6.3. Let $Q, M \geq 1$ and $C$ be a sufficiently large positive constant. Then we have the following inequalities:

$$
\begin{gathered}
C_{2}(M, Q) \ll(Q M)^{\varepsilon}\left(M+Q^{7 / 4}\right) ; \\
C_{2}(M, Q) \ll M^{\varepsilon} Q^{1-1 / v} \sum_{j=0}^{v-1} C_{2}\left(2^{j} M^{v}, Q\right)^{1 / v}, \quad \text { for each fixed positive integer } v \\
B_{1}\left(Q_{1}, M\right) \ll B_{1}\left(Q_{2}, M\right), \quad \text { if } Q_{1}, M \geq 1 \text { and } Q_{2} \geq C Q_{1} \log \left(2 Q_{1} M\right) ; \\
B_{2}(Q, M) \ll(\log 2 Q)^{3} Q^{1 / 2} X^{-1 / 2} B_{1}\left(X Q^{\varepsilon}, M\right), \quad \text { for some } X \text { with } 1 \leq X \leq Q ; \\
B_{3}(Q, M) \ll(\log 2 Q)^{3} Q^{1 / 2} X^{-1 / 2} B_{2}\left(X Q^{\varepsilon}, M\right), \quad \text { for some } X \text { with } 1 \leq X \leq Q ; \\
B_{3}(Q, M) \ll M^{\varepsilon} B_{4}\left(\frac{Q}{\Delta_{1}}, \frac{M}{\Delta_{2}}\right), \quad \text { for some } \Delta_{1}, \Delta_{2} \in \mathbb{N} \text { with } \Delta_{2}^{2} \geq \Delta_{1} ; \\
B_{4}(Q, M) \ll Q+Q M^{\varepsilon-2} \max \left\{B_{3}(K, M): K \leq M^{4} Q^{-1}\right\}+Q^{-1} M^{6+\varepsilon} \sum_{K>M^{4} / Q} K^{-2-\varepsilon} B_{3}(K, M),
\end{gathered}
$$

where the sum over $K$ in (6.11) runs over powers of 2 . 
Since the proofs of (6.6)-(6.11) are essentially the same as those of (31)-(36) of Lemma 4.1 in [3], we omit the proofs here.

We note that it follows from (6.3)-(6.6), that we have

$$
B_{1}(Q, M) \ll(Q M)^{\varepsilon}\left(Q^{1-1 / v} M+Q^{1+3 /(4 v)}\right)
$$

for any $v \in \mathbb{N}$.

6.4. Estimating $C_{2}$. In this section we prove (6.5). Recall $C_{2}(M, Q)$ is the norm of the sum

$$
\sum_{M<m \leq 2 M}\left|\sum_{\substack{n \in \mathbb{Z}[i] \\ Q<\mathcal{N}(n) \leq 2 Q \\ n \equiv \pm 1 \bmod (1+i)^{3}}}^{\prime} b_{n} \chi_{n}(m)\right|^{2},
$$

where the apostrophe indicates that $n$ is square-free and has no rational prime divisor.

The sum in (6.13) is obviously bounded by

$$
\ll \sum_{m \in \mathbb{Z}} W\left(\frac{m}{M}\right)\left|\sum_{n}^{\prime} b_{n} \chi_{n}(m)\right|^{2},
$$

where the weight function $W$ is defined as in (3.5). Expanding out the sum in (6.14) we get

$$
\sum_{\substack{n_{1}, n_{2} \in \mathbb{Z}[i] \\ Q<\mathcal{N}\left(n_{1}\right), \mathcal{N}\left(n_{2}\right) \leq 2 Q \\ n_{1}, n_{2} \equiv \pm 1 \bmod (1+i)^{3}}}^{\prime} b_{n_{1}} \bar{b}_{n_{2}} \sum_{m \in \mathbb{Z}} W\left(\frac{m}{M}\right) \chi_{n_{1}} \bar{\chi}_{n_{2}}(m) .
$$

Now we extract the greatest common divisor $\Delta$ of $n_{1}$ and $n_{2}$, getting

$$
\sum_{\substack{\mathcal{N}(\Delta) \leq 2 Q \\ \Delta \equiv 1 \bmod (1+i)^{3}}} \sum_{\substack{Q \\ \frac{Q}{\mathcal{N}(\Delta)}<\mathcal{N}\left(n_{1}\right), \mathcal{N}\left(n_{2}\right) \leq \frac{2 Q}{\mathcal{N}(\Delta)} \\ n_{1}, n_{2} \equiv \equiv \bmod (1+i)^{3} \\\left(n_{1}, n_{2}\right)=1 \\\left(n_{1} n_{2}, \Delta \bar{\Delta}\right)=1}}^{\prime} b_{n_{1} \Delta} \bar{b}_{n_{2} \Delta} \sum_{\substack{m \in \mathbb{Z} \\(m, \mathcal{N}(\Delta))=1}} W\left(\frac{m}{M}\right) \chi_{n_{1}} \bar{\chi}_{n_{2}}(m) .
$$

It is easy to see that there is a one-to-one correspondence between the pairs $\left(n_{1}, n_{2}\right)$ and $\left(n_{1}^{\prime} \Delta, n_{2}^{\prime} \Delta\right)$ with $\Delta \equiv 1, n_{1} \equiv n_{1}^{\prime}$ and $n_{2} \equiv n_{2}^{\prime} \bmod (1+i)^{3}$. We write $\delta=\left(n_{1}, \bar{n}_{2}\right)$ and change variables via $n_{1} \rightarrow \delta n_{1}$, $n_{2} \rightarrow \bar{\delta} n_{2}$ to get

$$
\begin{aligned}
& \sum_{\substack{\mathcal{N}(\Delta) \leq 2 Q \\
\Delta \equiv 1 \bmod (1+i)^{3}}}^{\prime} \sum_{\substack{\mathcal{N}(\delta) \leq \frac{2 Q}{\mathcal{N}(\Delta)} \\
\delta \equiv 1 \bmod (1+i)^{3}}}^{\prime} \sum_{\substack{Q \\
\mathcal{N}(\delta \Delta)}}^{\prime} \sum_{\substack{n_{1}, n_{2} \in \mathbb{N}[i] \\
\left(n_{1}\right), \mathcal{N}\left(n_{2}\right) \leq \frac{2 Q}{\mathcal{N}(\delta \Delta)}}}^{\prime} b_{n_{1} \Delta \delta} \bar{b}_{n_{2} \Delta \bar{\delta}} \\
& \begin{array}{cc}
(\mathcal{N}(\delta), \mathcal{N}(\Delta))=1 & n_{1}, n_{2} \equiv \pm 1 \bmod (1+i)^{3} \\
\left(\mathcal{N}\left(n_{1}\right), \mathcal{N}\left(n_{2}\right)\right)=1
\end{array} \\
& \left(n_{1} n_{2}, \delta \bar{\delta} \Delta \bar{\Delta}\right)=1 \\
& \times \sum_{\substack{m \in \mathbb{Z} \\
(m, \mathcal{N}(\Delta))=1}} W\left(\frac{m}{M}\right) \chi_{n_{1}} \bar{\chi}_{n_{2}} \bar{\chi}_{\delta}^{2}(m),
\end{aligned}
$$

where we use that for $m \in \mathbb{Z}, \chi_{\delta} \bar{\chi}_{\bar{\delta}}(m)=\chi_{\delta}^{2}(m)=\bar{\chi}_{\delta}^{2}(m)$. Next we remove the coprimality condition in the sum over $m$ by the Möbius function, getting

$$
\sum_{\substack{m \in \mathbb{Z} \\(m, \mathcal{N}(\Delta))=1}} W\left(\frac{m}{M}\right) \chi_{n_{1}} \bar{\chi}_{n_{2}} \bar{\chi}_{\delta}^{2}(m)=\sum_{\ell \mid \mathcal{N}(\Delta)} \mu(\ell) \chi_{n_{1}} \bar{\chi}_{n_{2}} \bar{\chi}_{\delta}^{2}(\ell) \sum_{m \in \mathbb{Z}} W\left(\frac{m}{M / \ell}\right) \chi_{n_{1}} \bar{\chi}_{n_{2}} \bar{\chi}_{\delta}^{2}(m),
$$


which by the Poisson summation formula is

$$
\begin{aligned}
\sum_{\ell \mid \mathcal{N}(\Delta)} \mu(\ell) \chi_{n_{1}} \bar{\chi}_{n_{2}} \bar{\chi}_{\delta}^{2}(\ell) & \frac{M}{\ell \mathcal{N}\left(n_{1} n_{2} \delta\right)} \sum_{h \in \mathbb{Z}} \widehat{W}\left(\frac{h M}{\ell \mathcal{N}\left(n_{1} n_{2} \delta\right)}\right) \\
& \times \sum_{r \bmod \mathcal{N}\left(n_{1} n_{2} \delta\right)} \chi_{n_{1}} \bar{\chi}_{n_{2}} \bar{\chi}_{\delta}^{2}(r) e\left(\frac{r h}{\mathcal{N}\left(n_{1} n_{2} \delta\right)}\right),
\end{aligned}
$$

where

$$
\widehat{W}(x)=\int_{-\infty}^{\infty} W(y) e(-x y) d y .
$$

When $h=0$, the expression in (6.16) vanishes unless $n_{1}=n_{2}=\delta=1$. Hence, the contribution of $h=0$ to (6.15) is

$$
\ll M Q^{\varepsilon} \sum_{\substack{Q<\mathcal{N}(\Delta) \leq 2 Q \\ \Delta \equiv 1 \bmod (1+i)^{3}}}^{\prime}\left|b_{\Delta}\right|^{2} \ll M Q^{\varepsilon}\left\|b_{n}\right\|^{2} .
$$

In the sequel, we assume that $h \neq 0$. The sum over $r$ in (6.16) can be computed by writing $r=$ $r_{1} \mathcal{N}\left(n_{2} \delta\right)+r_{2} \mathcal{N}\left(n_{1} \delta\right)+r_{3} \mathcal{N}\left(n_{1} n_{2}\right)$ to get

$$
\begin{aligned}
& \sum_{r \bmod \mathcal{N}\left(n_{1} n_{2} \delta\right)} \chi_{n_{1}} \bar{\chi}_{n_{2}} \bar{\chi}_{\delta}^{2}(r) e\left(\frac{r h}{\mathcal{N}\left(n_{1} n_{2} \delta\right)}\right) \\
&= \sum_{r_{1} \bmod \mathcal{N}\left(n_{1}\right)} \chi_{n_{1}}\left(r_{1} \mathcal{N}\left(n_{2} \delta\right)\right) e\left(\frac{r_{1} h}{\mathcal{N}\left(n_{1}\right)}\right) \sum_{r_{2} \bmod \mathcal{N}\left(n_{2}\right)} \bar{\chi}_{n_{2}}\left(r_{2} \mathcal{N}\left(n_{1} \delta\right)\right) e\left(\frac{r_{2} h}{\mathcal{N}\left(n_{2}\right)}\right) \\
& \times \sum_{r_{3} \bmod \mathcal{N}(\delta)} \bar{\chi}_{\delta}^{2}\left(r_{3} \mathcal{N}\left(n_{1} n_{2}\right)\right) e\left(\frac{r_{3} h}{\mathcal{N}(\delta)}\right) \\
&=\bar{\chi}_{n_{1}}(h) \chi_{n_{2}} \chi_{\delta}^{2}(-h) \chi_{n_{1}}\left(\mathcal{N}\left(n_{2} \delta\right)\right) \bar{\chi}_{n_{2}}\left(\mathcal{N}\left(n_{1} \delta\right)\right) \bar{\chi}_{\delta}^{2}\left(\mathcal{N}\left(n_{1} n_{2}\right)\right) \tau\left(\chi_{n_{1}}\right) \overline{\tau\left(\chi_{n_{2}}\right) \tau\left(\chi_{\delta}^{2}\right)}
\end{aligned}
$$

where $\tau(\chi)$ is defined as in (2.2).

Using quartic reciprocity and the identity

$$
\left(\frac{m}{n}\right)_{4}=\overline{\left(\frac{\bar{m}}{\bar{n}}\right)_{4}}
$$

following from the definition of the quartic residue symbol, we get the identities

$$
\begin{aligned}
& \chi_{n}(\mathcal{N}(m)) \bar{\chi}_{m}(\mathcal{N}(n))=\left(\frac{\mathcal{N}(m)}{n}\right)_{4} \overline{\left(\frac{\mathcal{N}(n)}{m}\right)_{4}}=\left(\frac{m}{\bar{n}}\right)_{4}^{2}=\chi_{\bar{n}}^{2}(m) \\
& \chi_{n}(\mathcal{N}(m)) \bar{\chi}_{m}^{2}(\mathcal{N}(n))=\left(\frac{\mathcal{N}(m)}{n}\right)_{4}\left(\frac{\mathcal{N}(n)}{m}\right)_{4}^{2}=\left(\frac{\mathcal{N}(m)}{\bar{n}}\right)_{4}=\chi_{\bar{n}}(\mathcal{N}(m))
\end{aligned}
$$

and

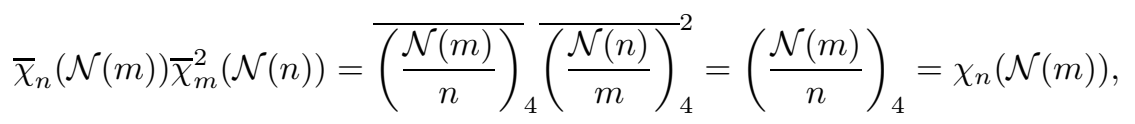

valid for all $m, n \in \mathbb{Z}[i]$ with $m, n \equiv \pm 1 \bmod (1+i)^{3}$. We use them to simplify the last line of (6.18), obtaining

$$
\bar{\chi}_{n_{1}}(h) \chi_{n_{2}} \chi_{\delta}^{2}(-h)\left(\frac{n_{2}^{2} \mathcal{N}(\delta)}{\overline{n_{1}}}\right)_{4}\left(\frac{\mathcal{N}(\delta)}{n_{2}}\right)_{4} \tau\left(\chi_{n_{1}}\right) \overline{\tau\left(\chi_{n_{2}}\right) \tau\left(\chi_{\delta}^{2}\right)} .
$$


Now, changing $n_{2} \rightarrow \bar{n}_{2}$, the contribution of $h \neq 0$ to the sum in (6.16) takes the form

$$
\begin{aligned}
& S_{W}(M, Q)=M \sum_{\substack{\mathcal{N}(\Delta) \leq 2 Q \\
\Delta \equiv 1 \bmod (1+i)^{3}}}^{\prime} \sum_{\substack{\mathcal{N}(\delta) \leq \frac{2 Q}{\mathcal{N}(\Delta)} \\
\left(\mathcal{N}(\delta), \mathcal{N}(\Delta)+(1)^{3}\right.}}^{\prime} \frac{\overline{\tau\left(\chi_{\delta}\right)}}{\mathcal{N}(\delta)} \sum_{\ell \mid \mathcal{N}(\Delta)} \frac{\mu(\ell)}{\ell} \bar{\chi}_{\delta}^{2}(\ell) \sum_{h \neq 0} \chi_{\delta}^{2}(h) \\
& \times \quad \sum_{n_{1}, n_{2} \in \mathbb{Z}[i]}^{\prime} \quad \widehat{W}\left(\frac{h M}{\ell \mathcal{N}\left(n_{1} n_{2} \delta\right)}\right) c_{\Delta, \delta, \ell, h, n_{1}} c_{\Delta, \delta, \ell, h, n_{2}}^{\prime}\left(\frac{n_{1}}{n_{2}}\right)_{4}^{2}, \\
& \frac{Q}{\mathcal{N}(\delta \Delta)}<\mathcal{N}\left(n_{1}\right), \mathcal{N}\left(n_{2}\right) \leq \frac{2 Q}{\mathcal{N}(\delta \Delta)} \\
& n_{1}, n_{2} \equiv \pm 1 \bmod (1+i)^{3} \\
& \left(\mathcal{N}\left(n_{1}\right), \mathcal{N}\left(n_{2}\right)\right)=1 \\
& \left(n_{1} n_{2}, \delta \bar{\delta} \Delta \bar{\Delta}\right)=1
\end{aligned}
$$

where

$$
c_{\Delta, \delta, \ell, h, n}:=\chi_{n}(\ell) \bar{\chi}_{n}(h)\left(\frac{\mathcal{N}(\delta)}{\bar{n}}\right)_{4} \frac{\tau\left(\chi_{n}\right)}{\mathcal{N}(n)} b_{n \Delta \delta} \quad \text { and } \quad c_{\Delta, \delta, \ell, h, n}^{\prime}:=\chi_{n}(\ell) \bar{\chi}_{n}(h)\left(\frac{\mathcal{N}(\delta)}{\bar{n}}\right)_{4} \frac{\tau\left(\chi_{n}\right)}{\mathcal{N}(n)} \bar{b}_{\bar{n} \Delta \bar{\delta}} .
$$

We now estimate the sum over $n_{1}$ and $n_{2}$ directly using (1.3). We denote the inner sum in the definition of $S_{W}(M, Q)$ above to be $U(\Delta, \delta, \ell, h)$ so that

$$
\begin{aligned}
& U(\Delta, \delta, \ell, h)=\quad \sum_{n_{1}, n_{2} \in \mathbb{Z}[i]}^{\prime} \quad \widehat{W}\left(\frac{h M}{\ell \mathcal{N}\left(n_{1} n_{2} \delta\right)}\right) c_{\Delta, \delta, \ell, h, n_{1}} c_{\Delta, \delta, \ell, h, n_{2}}^{\prime}\left(\frac{n_{1}}{n_{2}}\right)_{4}^{2} . \\
& \frac{Q}{\mathcal{N}(\delta \Delta)}<\mathcal{N}\left(n_{1}\right), \mathcal{N}\left(n_{2}\right) \leq \frac{2 Q}{\mathcal{N}(\delta \Delta)} \\
& n_{1}, n_{2} \equiv \pm 1 \bmod (1+i)^{3} \\
& \left(\mathcal{N}\left(n_{1}\right), \mathcal{N}\left(n_{2}\right)\right)=1 \\
& \left(n_{1} n_{2}, \delta \bar{\delta} \Delta \bar{\Delta}\right)=1
\end{aligned}
$$

To separate the variables $n_{1}, n_{2}$, we remove the coprimality condition $\left(\mathcal{N}\left(n_{1}\right), \mathcal{N}\left(n_{2}\right)\right)=1$. Because of the presence of $\left(\frac{n_{1}}{n_{2}}\right)_{4}$, we may assume that $n_{1}$ and $n_{2}$ are coprime. Thus $\mathcal{N}\left(n_{1}\right)$ and $\mathcal{N}\left(n_{2}\right)$ must also be coprime unless $n_{1}$ has a factor in common with $\bar{n}_{2}$. We proceed to detect this latter condition using the Mobius function in the standard way to obtain

$$
\begin{aligned}
& U(\Delta, \delta, \ell, h)=\sum_{\substack{e \in \mathbb{Z}[i] \\
e \equiv 1 \bmod (1+i)^{3} \\
(\mathcal{N}(e), \mathcal{N}(\delta \Delta))=1}}^{\prime} \mu_{[i]}(e) \sum_{\substack{n_{1}, n_{2} \in \mathbb{Z}[i] \\
\frac{Q}{\mathcal{N}(\delta \Delta)}<\mathcal{N}\left(n_{1}\right), \mathcal{N}\left(n_{2}\right) \leq \frac{2 Q}{\mathcal{N}(\delta \Delta)} \\
n_{1}, n_{2} \equiv \pm 1 \bmod (1+i)^{3} \\
e\left|n_{1}, \bar{e}\right| n_{2} \\
\left(n_{1} n_{2}, \delta \bar{\delta} \Delta \bar{\Delta}\right)=1}}^{\prime} \widehat{W}\left(\frac{h M}{\ell \mathcal{N}\left(n_{1} n_{2} \delta\right)}\right) c_{\Delta, \delta, \ell, h, n_{1}} c_{\Delta, \delta, \ell, h, n_{2}}^{\prime}\left(\frac{n_{1}}{n_{2}}\right)_{4}^{2} \\
& =\sum_{\substack{e \in \mathbb{Z}[i] \\
e \equiv 1 \bmod (1+i)^{3} \\
\mathcal{N}(e) \leq \frac{2 Q}{\mathcal{N}(\delta)} \\
(\mathcal{N}(e), \mathcal{N}(\delta \Delta))=1}}^{\prime} \mu_{[i]}(e) \chi_{\mathcal{N}(e)}(\ell) \bar{\chi}_{\mathcal{N}(e)}(h)\left(\frac{\mathcal{N}(\delta)}{\mathcal{N}(e)}\right)_{4} \frac{\tau\left(\chi_{e}\right)}{\mathcal{N}(e)} \frac{\tau\left(\chi_{\bar{e}}\right)}{\mathcal{N}(\bar{e})}\left(\frac{e}{\bar{e}}\right)_{4}^{2} \\
& \times \quad \sum_{n_{1}, n_{2} \in \mathbb{Z}[i]}^{\prime} \widehat{W}\left(\frac{h M}{\ell \mathcal{N}\left(n_{1} n_{2} e \bar{e} \delta\right)}\right) c_{\Delta, \delta, \ell, h, e, n_{1}} c_{\Delta, \delta, \ell, h, e, n_{2}}^{\prime}\left(\frac{n_{1}}{n_{2}}\right)_{4}^{2} . \\
& \frac{Q}{\mathcal{N}(e \delta \Delta)}<\mathcal{N}\left(n_{1}\right), \mathcal{N}\left(n_{2}\right) \leq \frac{2 Q}{\mathcal{N}(e \delta \Delta)} \\
& n_{1}, n_{2} \equiv \pm 1 \bmod (1+i)^{3} \\
& \left(n_{1} n_{2}, e \bar{e} \delta \bar{\delta} \Delta \bar{\Delta}\right)=1
\end{aligned}
$$

where

$$
c_{\Delta, \delta, \ell, h, e, n}:=\chi_{n}(\ell) \bar{\chi}_{n}(h)\left(\frac{\mathcal{N}(\delta)}{\bar{n}}\right)_{4}\left(\frac{\mathcal{N}(e)}{n}\right)_{4}\left(\frac{\mathcal{N}(n)}{e}\right)_{4}\left(\frac{n_{1}}{\bar{e}}\right)_{4}^{2} \frac{\tau\left(\chi_{n}\right)}{\mathcal{N}(n)} b_{n e \Delta \delta}
$$

and

$$
c_{\Delta, \delta, \ell, h, e, n}^{\prime}:=\chi_{n}(\ell) \bar{\chi}_{n}(h)\left(\frac{\mathcal{N}(\delta)}{\bar{n}}\right)_{4}\left(\frac{\mathcal{N}(e)}{n}\right)_{4}\left(\frac{\mathcal{N}(n)}{\bar{e}}\right)_{4}\left(\frac{e}{n_{2}}\right)_{4}^{2} \frac{\tau\left(\chi_{n}\right)}{\mathcal{N}(n)} \bar{b}_{\bar{n} e \Delta \bar{\delta}} .
$$


Next observe that we may freely truncate the sum over $h$ for

$$
|h| \leq \frac{Q^{2} \ell}{\mathcal{N}(\delta) \mathcal{N}(\Delta)^{2} M}(Q M)^{\varepsilon}=: H
$$

since $\widehat{W}$ has rapid decay. More precisely, if we let $S_{W}(M, Q)=S_{W}^{\prime}(M, Q)+E$ where $S_{W}^{\prime}(M, Q)$ is the contribution to $S_{W}(M, Q)$ from $0<|h| \leq H$, then $E \ll(M Q)^{-100}\|b\|^{2}$.

It remains to bound $S_{W}^{\prime}(M, Q)$ and we have

$$
\begin{aligned}
& S_{W}^{\prime}(M, Q) \ll M \sum_{\substack{\mathcal{N}(\Delta) \leq 2 Q \\
\Delta \equiv 1 \bmod (1+i)^{3}}}^{\prime} \sum_{\substack{\mathcal{N}(\delta) \leq \frac{2 Q}{\mathcal{N}(\Delta)} \\
\delta \equiv 1 \bmod (1+i)^{3} \\
(\mathcal{N}(\delta), \mathcal{N}(\Delta))=1}}^{\prime} \frac{1}{(\mathcal{N}(\delta))^{1 / 2}} \sum_{\ell \mid \mathcal{N}(\Delta)} \frac{1}{\ell} \\
& \times \sum_{\substack{e \in \mathbb{Z}[i] \\
e \equiv 1 \bmod (1+i)^{3} \\
\mathcal{N}(e) \leq \frac{2 Q}{\mathcal{N}(\delta \Delta)} \\
(\mathcal{N}(e), \mathcal{N}(\delta \Delta))=1}}^{\prime} \frac{1}{\mathcal{N}(e)} \sum_{0<|h| \leq H}\left|U^{\prime}(\Delta, \delta, \ell, e, h)\right|,
\end{aligned}
$$

where

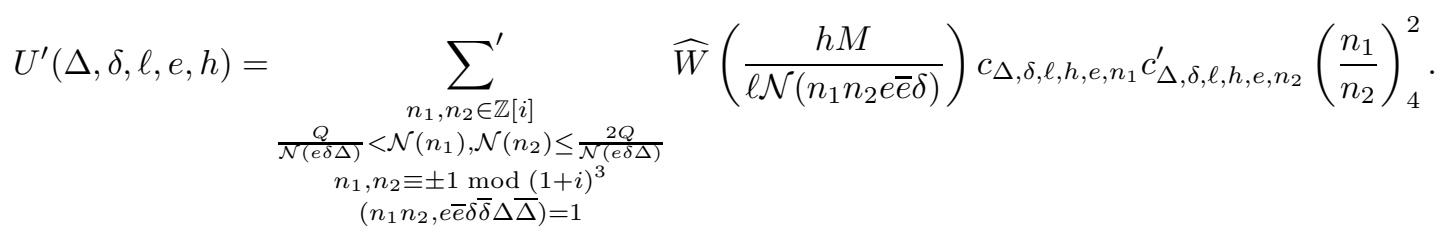

We now remove the weight $\widehat{W}$ in $U^{\prime}(\Delta, \delta, \ell, e, h)$ by applying Lemma 4.2 to the function $\rho(x)=\widehat{W}(x)$, which satisfies the conditions of that lemma, as one sees by repeated integration by parts. We may assume $h>0$ here, since the contribution of the negative $h$ 's can be treated similarly and satisfies the same bound. We use $\sigma=\varepsilon$ to see that

$$
U^{\prime}(\Delta, \delta, \ell, e, h) \ll_{\varepsilon}\left(\frac{\ell \mathcal{N}(e \bar{e} \Delta)}{h M}\right)^{\varepsilon} \int_{-\infty}^{\infty}\left|\rho_{+}(\varepsilon+i t)\right||V(\varepsilon+i t)| \mathrm{d} t,
$$

where

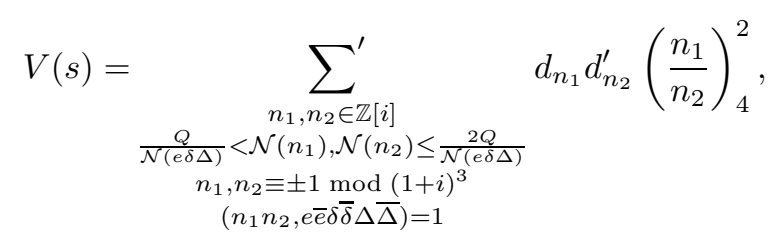

with

$$
d_{n}=c_{\Delta, \delta, \ell, h, e, n} \mathcal{N}(n)^{s} \text { and } d_{n}^{\prime}=c_{\Delta, \delta, \ell, h, e, n}^{\prime} \mathcal{N}(n)^{s} .
$$

Note that $d_{n_{1}}$ and $d_{n_{2}}^{\prime}$ depend on $\Delta, \delta, \ell, h, e, n$ and $s$, and

$$
\left|d_{n}\right| \ll\left(\frac{\mathcal{N}(e \delta \Delta)}{Q}\right)^{1 / 2-\varepsilon}\left|b_{n e \Delta \delta}\right|, \quad\left|d_{n}^{\prime}\right| \ll\left(\frac{\mathcal{N}(e \delta \Delta)}{Q}\right)^{1 / 2-\varepsilon}\left|b_{\bar{n} e \Delta \bar{\delta}}\right| .
$$

Now, using the Cauchy-Schwarz inequality and the estimate (1.3) upon noting that this estimate remains valid if the summation conditions $m, n \equiv 1 \bmod (1+i)^{3}$ therein are replaced by $m, n \equiv \pm 1 \bmod (1+i)^{3}$ and 
$\left(\frac{n}{m}\right)_{4}$ replaced by $\left(\frac{n_{1}}{n_{2}}\right)_{4}^{2}$, we bound $V(\varepsilon+i t)$ by

$$
\begin{aligned}
& |V(\varepsilon+i t)|^{2}
\end{aligned}
$$

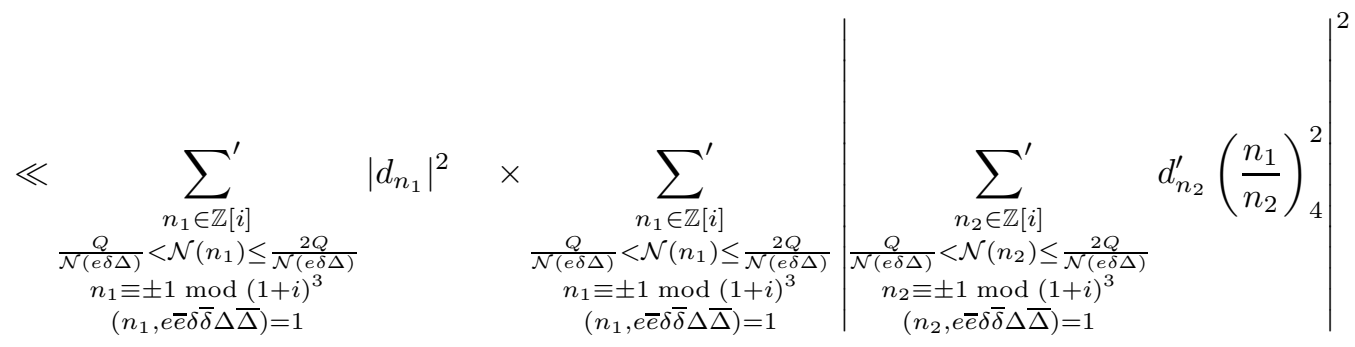

$$
\begin{aligned}
& \ll(Q M)^{8 \varepsilon}\left(\frac{\mathcal{N}(e \delta \Delta)}{Q}\right)^{1 / 4-4 \varepsilon}\left(\sum_{\substack{Q / \mathcal{N}(e)<\mathcal{N}(n) \leq 2 Q / \mathcal{N}(e) \\
n \equiv \pm 1 \bmod (1+i)^{3} \\
(\mathcal{N}(n), \mathcal{N}(e))=1}}^{\prime}\left|b_{e n}\right|^{2}\right)^{2}
\end{aligned}
$$

Since

$$
\int_{-\infty}^{\infty}\left|\rho_{+}(\sigma+i t)\right| \mathrm{d} t \ll_{\varepsilon} 1,
$$

we deduce that

$$
\begin{aligned}
& S_{W}^{\prime}(M, Q) \ll M(Q M)^{7 \varepsilon} \sum_{\substack{\mathcal{N}(\Delta) \leq 2 Q \\
\Delta \equiv 1 \bmod (1+i)^{3}}}^{\prime} \sum_{\substack{\mathcal{N}(\delta) \leq \frac{2 Q}{\mathcal{N}(\Delta)} \\
\delta \equiv 1 \bmod (1+i)^{3} \\
(\mathcal{N}(\delta), \mathcal{N}(\Delta))=1}}^{\prime} \frac{1}{(\mathcal{N}(\delta))^{1 / 2}} \sum_{\ell \mid \mathcal{N}(\Delta)} \frac{1}{\ell} \sum_{\substack{e \in \mathbb{Z}[i] \\
e \equiv 1 \bmod (1+i)^{3} \\
\mathcal{N}(e) \leq \frac{2 Q}{\mathcal{N}(\Delta)} \\
(\mathcal{N}(e), \mathcal{N}(\delta \Delta))=1}}^{\prime} \frac{1}{\mathcal{N}(e)} \\
& \times \sum_{0<|h| \leq H}\left(\frac{\mathcal{N}(e \delta \Delta)}{Q}\right)^{\frac{1}{4}-2 \varepsilon} \sum_{\substack{Q / \mathcal{N}(e)<\mathcal{N}(n) \leq 2 Q / \mathcal{N}(e) \\
n \equiv \pm 1 \bmod (1+i)^{3} \\
(\mathcal{N}(n), \mathcal{N}(e))=1}}^{\prime}\left|b_{e n}\right|^{2} \\
& \ll Q^{7 / 4+2 \varepsilon}(Q M)^{8 \varepsilon} \sum_{\substack{\mathcal{N}(\Delta) \leq 2 Q \\
\Delta \equiv 1 \bmod (1+i)^{3}}}^{\prime} \frac{1}{\mathcal{N}(\Delta)^{7 / 4+2 \varepsilon}} \sum_{\substack{\mathcal{N}(\delta) \leq \frac{2 Q}{\mathcal{N}(\Delta)} \\
\delta \equiv 1 \bmod (1+i)^{3} \\
(\mathcal{N}(\delta), \mathcal{N}(\Delta))=1}}^{\prime} \frac{1}{(\mathcal{N}(\delta))^{5 / 4+2 \varepsilon}} \sum_{\ell \mid \mathcal{N}(\Delta)} 1 \\
& \times \sum_{\substack{e \in \mathbb{Z}[i] \\
e \equiv 1 \bmod (1+i)^{3} \\
\mathcal{N}(e) \leq \frac{2 Q}{\mathcal{N}(\delta \Delta)}}}^{\prime} \frac{1}{\mathcal{N}(e)^{3 / 4+2 \varepsilon}} \sum_{\substack{Q / \mathcal{N}(e)<\mathcal{N}(n) \leq 2 Q / \mathcal{N}(e) \\
n \equiv \pm 1 \bmod (1+i)^{3} \\
(\mathcal{N}(n), \mathcal{N}(e))=1}}^{\prime}\left|b_{e n}\right|^{2} \\
& (\mathcal{N}(e), \mathcal{N}(\delta \Delta))=1 \\
& \ll Q^{7 / 4+2 \varepsilon}(Q M)^{8 \varepsilon} \sum_{\substack{Q<\mathcal{N}(n) \leq 2 Q \\
n \equiv \pm 1 \bmod (1+i)^{3}}}^{\prime}\left|b_{n}\right|^{2} \sum_{\substack{e \in \mathbb{Z}[i] \\
e \equiv 1 \bmod (1+i)^{3} \\
e \mid n}}^{\prime} \frac{1}{\mathcal{N}(e)^{3 / 4+2 \varepsilon}} \\
& \ll Q^{7 / 4+2 \varepsilon}(Q M)^{9 \varepsilon} \sum_{\substack{Q<\mathcal{N}(n) \leq 2 Q \\
n \equiv \pm 1 \bmod (1+i)^{3}}}^{\prime}\left|b_{n}\right|^{2} .
\end{aligned}
$$

Combining (6.17) and (6.20), we deduce that (6.15) and hence (6.13) is bounded by

$$
\ll(Q M)^{\varepsilon}\left(M+Q^{7 / 4}\right)\left\|b_{n}\right\|^{2}
$$


which implies the desired bound (6.5).

\section{Completion of the proof of Theorem 1.2}

We start with (6.12) with any $v \geq 2$ (as one checks easily that $v=1$ does not lead to any improvement) as an initial estimate. From (6.8) and (6.12), it follows that

$$
B_{2}(Q, M) \ll(Q M)^{\varepsilon} Q^{1 / 2} X^{-1 / 2}\left(X^{1+3 /(4 v)}+X^{1-1 / v} M\right)
$$

for a suitable $X$ with $1 \ll X \ll Q$. The worst case is $X=Q$ which shows $B_{2}(Q, M)$ also satisfies (6.12). Repeating the argument, we have

$$
B_{3}(Q, M) \ll(Q M)^{\varepsilon}\left(Q^{1+3 /(4 v)}+Q^{1-1 / v} M\right) .
$$

Combining this with (6.11), we obtain

$$
\begin{gathered}
B_{4}(Q, M) \ll Q+(Q M)^{9 \varepsilon} Q M^{-2} \max \left\{K^{1+3 /(4 v)}+K^{1-1 / v} M: K \leq M^{4} Q^{-1}\right\} \\
+(Q M)^{9 \varepsilon} M^{6} Q^{-1} \sum_{K \geq M^{4} / Q} K^{-2-\varepsilon}\left(K^{1+3 /(4 v)}+K^{1-1 / v} M\right) \\
\ll Q+(Q M)^{10 \varepsilon}\left(Q^{-3 /(4 v)} M^{2+3 / v}+Q^{1 / v} M^{3-4 / v}\right),
\end{gathered}
$$

where the sum over $K$ runs over powers of 2. From this and (6.10), we deduce that

$$
B_{3}(Q, M) \ll \frac{Q}{\Delta_{1}}+(Q M)^{\varepsilon}\left(\left(\frac{Q}{\Delta_{1}}\right)^{-3 /(4 v)}\left(\frac{M}{\Delta_{2}}\right)^{2+3 / v}+\left(\frac{Q}{\Delta_{1}}\right)^{1 / v}\left(\frac{M}{\Delta_{2}}\right)^{3-4 / v}\right)
$$

for some positive integers $\Delta_{1}, \Delta_{2}$ with $\Delta_{2}^{2} \geq \Delta_{1}$. From this and the trivial bound

$$
B_{1}(Q, M) \ll B_{3}(Q, M)
$$

we deduce that

$$
B_{1}(Q, M) \ll Q+(Q M)^{\varepsilon}\left(Q^{-3 /(4 v)} M^{2+3 / v}+Q^{1 / v} M^{3-4 / v}\right) .
$$

Combining (7.1) with (6.7), we deduce that

$$
B_{1}(Q, M) \ll(\tilde{Q} M)^{\varepsilon}\left(\tilde{Q}+\tilde{Q}^{-3 /(4 v)} M^{2+3 / v}+\tilde{Q}^{1 / v} M^{3-4 / v}\right)
$$

if $\tilde{Q} \geq C Q \log (2 Q M)$. We choose $\tilde{Q}:=\max \left(Q^{1+\varepsilon}, M^{4-4 v / 7}\right)$. Then (17.2) implies that

$$
B_{1}(Q, M) \ll(Q M)^{\varepsilon}\left(Q+Q^{1 / v} M^{3-4 / v}+M^{17 / 7}\right) .
$$

It's easy to see that the choice $v=2$ is optimal and a further cycle in the above process does not lead to an improvement of our result. Combining (6.12) with $v=1,2,3$ and (7.3) with $v=2$, we obtain our final estimate

$$
B_{1}(Q, M) \ll(Q M)^{\varepsilon} \min \left\{Q^{7 / 4}+M, Q^{11 / 8}+Q^{1 / 2} M, Q^{5 / 4}+Q^{2 / 3} M, Q+Q^{1 / 2} M+M^{17 / 7}\right\} .
$$

which together with (6.1) (noting that the last expression in (6.1) is $\ll B_{1}(Q, M)$ by the law of quartic reciprocity) implies Theorem 1.2 
Calculating the right-hand side of (1.4) for various ranges of $Q$ and $M$, we obtain that it is bounded by

$$
\ll(Q M)^{\varepsilon}\left\|a_{m}\right\|^{2} \cdot \begin{cases}M & \text { if } Q \leq M^{4 / 7}, \\ Q^{7 / 4} & \text { if } M^{4 / 7}<Q \leq M^{4 / 5}, \\ Q^{1 / 2} M & \text { if } M^{4 / 5}<Q \leq M^{8 / 7}, \\ Q^{11 / 8} & \text { if } M^{8 / 7}<Q \leq M^{24 / 17}, \\ Q^{2 / 3} M & \text { if } M^{24 / 17}<Q \leq M^{12 / 7}, \\ Q^{5 / 4} & \text { if } M^{12 / 7}<Q \leq M^{68 / 35}, \\ M^{17 / 7} & \text { if } M^{68 / 35}<Q \leq M^{17 / 7}, \\ Q & \text { if } M^{17 / 7}<Q .\end{cases}
$$

For convenience, we enclose a table displaying the estimates for $B_{1}(Q, M)$ that we get for various ranges. This table should be read as follows. If the fractions $\alpha$ and $\beta$ are the $(n-1)$-th and $n$-th entries, respectively, in the first row, and the term $T$ is the $n$-th entry in the second row, then the estimate $B_{1}(Q, M) \ll(Q M)^{\varepsilon} T$ holds in the range $M^{\alpha}<Q \leq M^{\beta}$.

\begin{tabular}{|l||l|l|l|l|l|l|l|l|}
\hline Range & $4 / 7$ & $4 / 5$ & $8 / 7$ & $24 / 17$ & $12 / 7$ & $68 / 35$ & $17 / 7$ & $\infty$ \\
\hline Bounds & $M$ & $Q^{7 / 4}$ & $Q^{1 / 2}$ & $Q^{11 / 8}$ & $Q^{2 / 3}$ & $Q^{5 / 4}$ & $M^{17 / 7}$ & $Q$ \\
\hline
\end{tabular}

It can be easily checked that (6.12) with $v \geq 3$ does not lead to an improvement of (7.4).

Acknowledgments. During this work, P. G. was supported by postdoctoral research fellowships at Nanyang Technological University (NTU) and L. Z. by an AcRF Tier I Grant at NTU. Moreover, L. Z. was on a visiting stay at Max-Planck-Institut für Mathematik (MPIM) in Bonn and he wishes to thank MPIM for its financial support and hospitality during that pleasant and enjoyable period. Finally, we thank the anonymous referee for his/her many comments and suggestions.

\section{REFERENCES}

[1] S. Baier, The large sieve with quadratic amplitude, Funct. Approx. Comment. Math. 36 (2006), 33-43.

[2] _ On the large sieve with sparse sets of moduli, J. Ramanujan Math. Soc. 21 (2006), 279-295.

[3] S. Baier and M. P. Young, Mean values with cubic characters, J. Number Theory 130 (2010), no. 4, 879-903.

[4] S. Baier and L. Zhao, Large sieve inequalities for characters to powerful moduli, Int. J. Number Theory 1 (2005), no. 2, 265-279.

[5] _ An improvement for the large sieve for square moduli, J. Number Theory 128 (2008), no. 1, 154-174.

[6] E. Bombieri, On the large sieves, Mathematika 12 (1965), 201-225.

[7] E. Bombieri and H. Davenport, Some inequalities involving trigonometrical polynomials, Ann. Sc. Norm. Super. Pisa 23 (1969), 223-241.

[8] H. Davenport, Multiplicative Number Theory, Third edition, Graduate Texts in Mathematics, vol. 74, Springer-Verlag, Berlin, etc., 2000.

[9] H. Davenport and H. Halberstam, The values of a trigonometric polynomial at well spaced points, Mathematika 13 (1966), 91-96. Corrigendum and Addendum, Mathematika 14 (1967), 232-299.

[10] P. D. T. A. Elliott, On the mean value of $f(p)$, Proc. London Math. Soc. (3) 21 (1970), $28-96$.

[11] P. X. Gallagher, The large sieve, Mathematika 14 (1967), 14-20.

[12] D. R. Heath-Brown, A mean value estimate for real character sums, Acta Arith. 72 (1995), no. 3, $235-275$.

[13] _ Kummer's conjecture for cubic Gauss sums, Israel J. Math. 120 (2000), 97-124.

[14] D. R. Heath-Brown and S. J. Patterson, The distribution of Kummer sums at prime arguments, J. Reine Angew. Math. 310 (1979), 111-130.

[15] K. Ireland and M. Rosen, A Classical Introduction to Modern Number Theory, Second edition, Graduate Texts in Mathematics, Springer-Verlag, New York, 1990.

[16] J. V. Linnik, The large sieve, Doklady Akad. Nauk SSSR 36 (1941), 119-120.

[17] W. Luo, On Hecke L-series associated with cubic characters, Compos. Math. 140 (2004), no. 5, 1191-1196. 
[18] H. L. Montgomery, Topics in Multiplicative Number Theory, Lecture Notes in Mathematics, vol. 227, Spring-Verlag, Barcelona, etc., 1971.

[19] _ Topics in Multiplicative Number Theory, Lecture Notes in Mathematics, vol. 227, Spring-Verlag, Berlin, etc., 1971.

[20] - The analytic priciples of large sieve, Bull. Amer. Math. Soc. 84 (1978), no. 4, 547-567.

[21] H. L. Montgomery and R. C. Vaughan, The large sieve, Mathematika 20 (1973), 119-134.

[22] S. J Patterson, The distribution of general Gauss sums and similar arithmetic functions at prime arguments, Prod. London Math. Soc. (3) 54 (1987), no. 2, 193-215.

[23] G. Prakash and D. S. Ramana, The large sieve with quadratic amplitude, J. Number Theory 129 (2009), no. 2, $428-433$.

[24] D. Wolke, On the large sieve with primes, Acta Math. Acad. Sci. Hungar. 22 (1971/72), 239-247.

[25] L. Zhao, Large sieve inequalities for special characters to prime square moduli, Funct. Approx. Comment. Math. 32 (2004), 99-106.

[26] _ Large sieve inequality for characters to square moduli, Acta Arith. 112 (2004), no. 3, 297-308.

$[27]-$ An improvement of a large sieve inequality in high dimensions, Mathematika 52 (2005), 93-100.

[28] _ Large sieve inequalities with quadratic amplitudes, Monatsh. Math. 151 (2007), no. 2, 165-173.

Peng Gao

Div. of Math. Sci., School of Phys. \& Math. Sci.,

Nanyang Technological Univ., Singapore 637371

Email: penggao@ntu.edu.sg
Liangyi Zhao

Div. of Math. Sci., School of Phys. \& Math. Sci., Nanyang Technological Univ., Singapore 637371

Email: lzhao@pmail.ntu.edu.sg 